\title{
Multi-zone warm and cold clumpy absorbers in three Seyfert galaxies
}

\author{
C. Ricci ${ }^{1,2}$, V. Beckmann ${ }^{3}$, M. Audard ${ }^{1,2}$, and T. J.-L. Courvoisier ${ }^{1,2}$ \\ 1 ISDC Data Centre for Astrophysics, University of Geneva, ch. d'Ecogia 16, 1290 Versoix, Switzerland \\ e-mail: Claudio.Ricci@unige.ch \\ 2 Geneva Observatory, University of Geneva, ch. des Maillettes 51, 1290 Versoix, Switzerland \\ 3 APC, François Arago Centre, Université Paris Diderot, CNRS/IN2P3, 10 rue A. Domon et L. Duquet, 75205 Paris Cedex, France
}

Received 15 May 2009 / Accepted 5 May 2010

\begin{abstract}
Aims. We present the first detailed X-ray analysis of three active galactic nuclei, the Seyfert 1 galaxies UGC 3142 and ESO 140-43, and the Seyfert 2 galaxy ESO 383-18, to study the geometry and the physical characteristics of their absorbers.

Methods. High-quality XMM-Newton EPIC and RGS data were analyzed as well as Swift/XRT and BAT and INTEGRAL IBIS/ISGRI data to cover the 0.3-110 keV energy range. For ESO 140-43 also XMM-Newton/OM and Swift/UVOT data were used. We studied the variability of the three AGN on a time-scale of seconds using the EPIC/PN light curves, and the long-term time-scale variability of ESO 140-43 using two observations performed six months apart by XMM-Newton.

Results. The spectra of the three Seyfert galaxies present a "soft excess" at energies $E<2 \mathrm{keV}$ above a power law continuum that can be modeled by complex absorption, without any additional emission component. The X-ray sources in UGC 3142 and ESO 383-18 are absorbed by two layers of neutral material, with covering fractions $f_{1} \simeq 0.92$ and $f_{2} \simeq 0.57$ for UGC 3142 , and $f_{1} \simeq 0.97$ and $f_{2} \simeq 0.86$ for ESO 383-18. While the clumpy absorber could be part of a disk wind or of the broad line region for UGC 3142, for ESO 383-18 a clumpy torus plus Compton thin dust lanes are more likely. The spectra of ESO 140-43 can be well fitted with a power law absorbed by three clumpy ionized absorbers with different covering factors, column densities, and ionization parameters, likely part of a moving clumpy system, which could be a disk wind or the broad line region. The strong spectral and flux variability on a time-scale of six months seen in ESO 140-43 is likely due to changes in the moving absorbers. We were able to detect the variation of the covering factor of one of the three ionized absorbers on a kilo-seconds time-scale in the EPIC light-curve of ESO 140-43.
\end{abstract}

Key words. galaxies: general - X-rays: galaxies - X-rays: general - galaxies: active - galaxies: Seyfert

\section{Introduction}

Active galactic nuclei (AGN) are thought to be powered by accretion onto super massive black holes. In this picture the X-ray emission is produced by UV photons from the innermost edge of the accretion disk, which undergo multiple inverse Compton scatterings by a population of hot electrons located in a coronal region sandwiching the disk (Haardt \& Maraschi 1991, 1993). The spectrum in the X-rays can be well approximated by a power law with an exponential cut-off at energy $E_{\mathrm{C}} \simeq k T_{\mathrm{e}}$, where $T_{\mathrm{e}}$ is the temperature of the electrons. Besides the power law-like continuum, two prominent features generated by the reflection of the continuum are observed in the X-ray spectra of AGN: a neutral iron $\mathrm{K} \alpha$ line (at $6.4 \mathrm{keV}$ in the local reference frame) and a reflection hump which peaks at $E \simeq 30 \mathrm{keV}$ (Magdziarz \& Zdziarski 1995). Not all these components are clearly observed in all AGN (e.g., Soldi et al. 2005; Beckmann et al. 2004).

An alternative model for the accretion process onto black holes is that of clumpy accretion flows (e.g., Guilbert \& Rees 1988). Courvoisier \& Türler (2005) assume that the different elements (clumps) of the accretion flow have velocities that may differ substantially. As a consequence, collisions between these clumps will appear when the clumps are close to the central object, resulting in emission (Ishibashi \& Courvoisier 2009).
At optical energies, AGN are classified according to their emission lines into type 1 (with broad and narrow emission lines), and type 2 (with only narrow lines). A similar distinction between the two types can be done in the X-rays. Here, type 2 objects show absorption which supresses the soft X-ray spectrum. The dividing line between unabsorbed and absorbed AGN is often set at a hydrogen column density of $N_{\mathrm{H}}=10^{22} \mathrm{~cm}^{-2}$. $\mathrm{X}$-ray data show that most AGN unabsorbed in X-ray are optical Seyfert 1 type, and most, but not all, AGN that are absorbed belong to the Seyfert 2 group (e.g., Awaki et al. 1991). Antonucci (1993) proposed that the extinguishing region forms a torus around the central region. The presence of an absorbing torus guarantees anisotropic obscuration of the central region, so that the sources viewed face-on are type 1 , and those observed edgeon are type 2 (for which, although it is highly extinguished, the broad line region exists). AGN spectra are mainly affected by absorption at energies $\lesssim 2 \mathrm{keV}$, with the effect depending on whether the absorber is cold (neutral or weakly ionized), or warm (highly ionized). The geometry of the absorber also plays a major role. When it does not completely cover the source (partially covering absorber), some continuum leaks out and reaches the observer, resulting in an apparent excess over the continuum at higher energies. The Einstein satellite provided the first evidence that the absorbing gas can cover only a fraction of the line of sight towards the AGN (Reichert et al. 1985; Holt et al. 1980). 
Table 1. Positions (J2000), redshift and Galactic hydrogen column densities (from Dickey \& Lockman 1990) of the three sources analyzed in this work.

\begin{tabular}{llllll}
\hline \hline Object & Type & RA & Dec & $z$ & $\begin{array}{l}\text { Galactic } N_{\mathrm{H}} \\
{\left[10^{21} \mathrm{~cm}^{-2}\right]}\end{array}$ \\
\hline UGC 3142 & Seyfert 1 & $04^{\mathrm{h}} 43^{\mathrm{m}} 46.8^{\mathrm{s}}$ & $+^{\circ} 8^{\circ} 58^{\prime} 19^{\prime \prime}$ & 0.0217 & 1.9 \\
ESO 383-18 & Seyfert 2 & $13^{\mathrm{h}} 33^{\mathrm{m}} 26.3^{\mathrm{s}}$ & $-34^{\circ} 00^{\prime} 59^{\prime \prime}$ & 0.0124 & 0.42 \\
ESO 140-43 & Seyfert 1 & $18^{\mathrm{h}} 44^{\mathrm{m}} 54.0^{\mathrm{s}}$ & $-62^{\circ} 21^{\prime} 53^{\prime \prime}$ & 0.0141 & 0.73 \\
\hline
\end{tabular}

The partial covering scenario can also explain some of the observed X-ray variability, which could be due to rearrangements of the cloud distribution (e.g., Abrassart \& Czerny 2000).

The first evidence of absorption due to warm material came from studies of the Einstein X-ray spectrum of the QSO MR 2251-178 (Halpern 1984; Pan et al. 1990), and from AGN spectra taken with ROSAT (Turner et al. 1993). Since then, highly ionized absorbers have been observed in about half of the X-ray spectra of type 1 AGN, both Seyfert 1 (e.g., Reynolds 1997; George et al. 1998) and quasars (Piconcelli et al. 2005), with column densities up to $\gtrsim 10^{23} \mathrm{~cm}^{-2}$, and often consisting of several zones of ionized gas (e.g., Nandra et al. 1993; Pounds et al. 1994; Kaspi et al. 2002; Steenbrugge et al. 2005). For NGC 3783, Netzer et al. (2003) and Krongold et al. (2003) discussed the observed complexity in the context of several phases of absorbing gas with distinct temperatures and ionization states. Evidence of a multi-phase warm absorber was also recently reported by Longinotti et al. (2010). The observed absorption lines are often blue-shifted with respect to the optical emission lines, which implies that they are produced in an outflowing region, with mean velocities covering the range from hundreds to thousands of $\mathrm{km} \mathrm{s}^{-1}$ (Kaspi et al. 2002; Krongold et al. 2003; Blustin et al. 2005; McKernan et al. 2007; Blustin et al. 2007). Analyzing NGC 3516, Turner et al. (2005) discovered a high column absorber with a hydrogen column density of $N_{\mathrm{H}} \sim 10^{23} \mathrm{~cm}^{-2}$ and a ionization parameter of $\log \xi \sim 2$, covering about $50 \%$ of the continuum source. Changes in the covering fraction of the absorber could explain the spectral variability observed in this source (Turner et al. 2008). A complete review of the recent developments of absorption models can be found in Turner \& Miller (2009).

A soft $(E \lesssim 2 \mathrm{keV})$ excess over the power law component dominant at higher energies has been found in the X-ray spectra of many Seyfert galaxies (Saxton et al. 1993). The origin of the soft excess is still an open issue. In the past the soft excess was often associated with the high-energy tail of the thermal emission of the disk, but it recently has been shown that the temperature of the disk should be constant $(0.1-0.2 \mathrm{keV})$, regardless of the mass and luminosity of the AGN (Gierlinski \& Done 2004). This result implies that some other mechanism is at work, as the temperature of the disk should depend on both the mass of the black hole and the accretion rate. Three competing models have been brought forward to explain the soft X-ray excess: i) an additional Comptonization component (e.g., Dewangan et al. 2007); ii) ionized reflection (e.g. from the disk, e.g. Crummy et al. 2006); iii) complex and/or ionized absorption (e.g., Gierlinski \& Done 2004). Chevallier et al. (2006) examined these three main possibilities and showed that although the absorption model can successfully fit the data of several AGN, for many AGN the quality of the data does not allow a firm conclusion. Nevertheless, they favor a reflection model absorbed by a modest relativistic wind. Done \& Nayakshin (2007) agree that the excess is most probably due to partially ionized material moving at relativistic speeds close to the black hole. The two potential scenarios for this effect would be an accretion disk seen in reflection or a wind above the disk, seen in absorption. They show that the reflection model would require very specific ionization parameters, contrary to the disk wind model. Schurch \& Done (2008) pointed out that although the absorption model with partially ionized material can be physically interpreted as a radiatively driven accretion disk wind, these winds would require velocities of $\sim 0.9 \mathrm{c}$ to reproduce the soft X-ray excess. They conclude that if the soft excess is produced by absorption, it seems more likely that the material is clumpy and/or only partially covers the source rather than forming a continuous outflow.

The three AGN discussed here are part of the INTEGRAL AGN catalog (Beckmann et al. 2009). Because at the time of the catalog's compilation no absorption information was available for a number of sources, unpublished XMM-Newton and Swift/XRT data were used to constrain $N_{\mathrm{H}}$. The three Seyfert galaxies UGC 3142, ESO 140-43, and ESO 383-18 are the sources which showed particularly strong evidence for excess in the soft X-ray spectra, which requires more complex modeling of the absorption.

The nearby Seyfert 1 UGC 3142 was first detected in X-ray by ROSAT (Boller et al. 1992), and in hard X-ray by INTEGRAL (Bassani et al. 2006). The Seyfert 2 ESO 383-18 was detected in hard X-ray by INTEGRAL (Krivonos et al. 2007). The Seyfert 1 ESO 140-43, also known as Fairall 51, was detected for the first time in the X-rays by HEAO A-2 (Marshall et al. 1979), and subsequently by ROSAT (Boller et al. 1992). At hard X-rays ESO 140-43 was detected by INTEGRAL IBIS/ISGRI (Beckmann et al. 2007). Information on the three sources are reported in Table 1. We present here the first detailed X-ray spectral analysis of these three Seyfert AGN using XMM-Newton, Swift and INTEGRAL data. The data analysis is described in Sect. 2. In Sect. 3 we discuss the spectral modeling of the X-ray spectra, and we present the study of the X-ray and optical/UV variability in Sect. 4. In Sect. 5 we discuss the obtained results, and we present the conclusions in Sect. 6.

\section{Data analysis}

The journal of the observations is shown in Table 2.

\subsection{XMM-Newton EPIC, RGS and OM}

The Seyfert 1 UGC 3142 was observed by the European Photon Imaging Camera (EPIC) on board the XMM-Newton space observatory (Jansen et al. 2001) in March 2007. The EPIC observation was performed with the PN camera (Struder et al. 2001) and the MOS (1 and 2) cameras (Turner et al. 2001), all of them operating in prime full window mode, and all equipped with the "medium" blocking filter.

The Seyfert 2 ESO 383-18 was observed by XMM-Newton in October 2006 with the three EPIC cameras operating in Prime Full Window mode. The PN and MOS 1 cameras were used with 
Table 2. X-ray observation log.

\begin{tabular}{|c|c|c|c|c|c|c|}
\hline$\overline{\overline{\text { Object }}}$ & Mission & $\overline{\text { Instrument }}$ & $\begin{array}{l}\text { Start time } \\
{[\mathrm{UT}]}\end{array}$ & $\begin{array}{l}\text { End time } \\
{[\mathrm{UT}]}\end{array}$ & $\begin{array}{l}\text { Exposure } \\
{[\mathrm{ks}]}\end{array}$ & 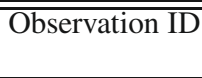 \\
\hline \multirow[t]{4}{*}{ UGC 3142} & INTEGRAL & IBIS/ISGRI & February 2003 & October 2008 & 485 & - \\
\hline & XMM-Newton & EPIC/PN & 2007-03-18 21:28:29 & 2007-03-19 00:15:51 & 8.5 & 0401790101 \\
\hline & XMM-Newton & EPIC/MOS & 2007-03-18 21:06:10 & 2007-03-19 00:19:51 & 11.6 & 0401790101 \\
\hline & XMM-Newton & RGS & 2007-03-18 21:05:31 & 2007-03-19 00:23:59 & 11.9 & 0401790101 \\
\hline \multirow[t]{4}{*}{ ESO 383-18 } & INTEGRAL & IBIS/ISGRI & March 2003 & August 2007 & 260 & - \\
\hline & XMM-Newton & EPIC/PN & 2006-01-10 02:49:18 & 2006-01-10 06:50:06 & 14.5 & 0307000901 \\
\hline & XMM-Newton & EPIC/MOS & 2006-01-10 02:26:59 & 2006-01-10 06:55:28 & 16.1 & 0307000901 \\
\hline & XMM-Newton & RGS & 2006-01-10 02:26:21 & 2006-01-10 06:54:50 & 16.1 & 0307000901 \\
\hline \multirow{9}{*}{ ESO $140-43$} & INTEGRAL & IBIS/ISGRI & 2007-10-29 & 2007-04-11 & 379 & - \\
\hline & XMM-Newton & EPIC/PN & 2005-09-08 03:09:46 & 2005-09-08 10:07:29 & 22.5 & 0300240401 \\
\hline & XMM-Newton & EPIC/MOS & 2005-09-08 02:47:28 & 2005-09-08 05:30:11 & 9.8 & 0300240401 \\
\hline & XMM-Newton & RGS & 2005-09-08 02:46:44 & 2005-09-08 10:15:22 & 26.9 & 0300240401 \\
\hline & XMM-Newton & $\mathrm{EPIC} / \mathrm{PN}$ & 2006-03-07 12:36:40 & 2006-03-07 18:19:46 & 18 & 0300240901 \\
\hline & XMM-Newton & EPIC/MOS & 2006-03-07 12:14:22 & 2006-03-07 18:23:52 & 17.6 & 0300240901 \\
\hline & XMM-Newton & RGS & 2006-03-07 12:13:44 & 2006-03-07 18:27:15 & 22.4 & 0300240901 \\
\hline & Swift & XRT & 2008-05-20 12:42:01 & $2008-05-20 \quad 14: 25: 13$ & 6.2 & 00037809001 \\
\hline & Swift & XRT & 2008-05-25 11:33:00 & $2008-05-25$ 12:26:20 & 3.2 & 00037809002 \\
\hline
\end{tabular}

Table 3. Background and source radii used for the extraction of XMM-Newton/EPIC spectra.

\begin{tabular}{lllllll}
\hline \hline Object & Instrument & Source & Background & Instrument & Source & Background \\
\hline UGC 3142 & EPIC/PN & $34.5^{\prime \prime}$ & $48.1^{\prime \prime}$ & EPIC/MOS & $32.2^{\prime \prime}$ & $62^{\prime \prime}$ \\
ESO 383-18 & EPIC/PN & $37^{\prime \prime}$ & $43.6^{\prime \prime}$ & EPIC/MOS & $24.2^{\prime \prime}$ & $47.8^{\prime \prime}$ \\
ESO 140-43 & EPIC/PN & $36.7^{\prime \prime}$ & $52.9^{\prime \prime}$ & EPIC/MOS & $37.5^{\prime \prime}$ & $66^{\prime \prime}$ \\
ESO 140-43 & EPIC/PN & $34^{\prime \prime}$ & $61.7^{\prime \prime}$ & EPIC/MOS & $26.5^{\prime \prime}$ & $50^{\prime \prime}$ \\
\hline
\end{tabular}

Notes. The index 1 refers to the 2005 observation of ESO 140-43, index 2 to the 2006 observation.

the "thin" blocking filter, while MOS 2 was using the "medium" filter.

The Seyfert 1 ESO 140-43 was observed twice by XMM-Newton. The first observation was performed in September 2005 (henceforth 2005-observation), while the second observation was carried out in March 2006 (henceforth 2006-observation). In both observations the three EPIC cameras were equipped with the "medium" blocking filter and operated in prime full mode.

We checked the background light curve in the $10-12 \mathrm{keV}$ energy band for PN and above $10 \mathrm{keV}$ for MOS in order to detect and filter the exposures for periods of high background activity. The recommended thresholds for the background count rate are $0.35 \mathrm{ct} / \mathrm{s}$ and $0.4 \mathrm{ct} / \mathrm{s}$ for the MOS and PN camera, respectively. For UGC 3142 only the PN is strongly affected, and the exposure is reduced from $10 \mathrm{ks}$ to $8.5 \mathrm{ks}$. In the case of ESO 383-18 this does not change anything in the good time intervals (GTI), as the background count rates of the observations are well below the recommended thresholds. Particular care has to be taken in the case of ESO 140-43, because the EPIC detectors experienced a strong flux of high-energy particles during the observations. During the 2005-observation this flux was between 0.4 and $2.2 \mathrm{ct} / \mathrm{s}$ for the PN, while it was much below the recommended threshold for MOS. The contribution of the background to the spectrum of the 2005-observation is weak $(\ll 1 \%)$ over the range considered in the analysis $(0.3-10 \mathrm{keV})$, thus we filtered only events with a background count rate higher than $1 \mathrm{ct} / \mathrm{s}$, which reduces the GTI to $22.5 \mathrm{ks}$, excluding the last $2.5 \mathrm{ks}$ of the observation. The 2006-observation presented a stronger flux of high-energy particles, with a maximum flux of $5 \mathrm{ct} / \mathrm{s}$ for PN and of $1.5 \mathrm{ct} / \mathrm{s}$ for MOS. In this observation the contribution of the background is not strong either $(\simeq 8 \%)$, and we set the background count rate threshold at 4 and $0.8 \mathrm{ct} / \mathrm{s}$ for the PN and MOS, respectively. This reduces the exposure time to $18 \mathrm{ks}$ for the PN, and to $17.6 \mathrm{ks}$ for the two MOS cameras.

We checked for pile-up with the epatplot task and found it to be negligible in all observations. For the analysis of EPIC data we used the XMM-Newton Standard Analysis Software (SAS) version 8.0.0, which also generates the ancillary response matrices (ARFs) and the detector response matrices (RMFs), through the tasks arfgen and rmfgen. We used the epchain and emchain tasks for processing the raw PN and MOS data files, respectively. We selected only patterns corresponding to single, double, triple, and quadruple events for EPIC/MOS (PATTERN $\leq 12$ ), while for EPIC/PN we considered only single and double events $($ PATTERN $\leq 4)$. The source spectra were extracted from the final filtered event list using circular regions centered on the sources, while the background was estimated from regions close to the source (on the same CCD), where no source was present. The same regions were used to estimate the PN light curves of the sources, which were corrected for the influence of the background with the task epiclccorr. The radii of these regions are listed in Table 3. The spectra obtained were grouped to have at least 20 counts per bin, in order to use $\chi^{2}$ statistics.

The RGS spectra were extracted with the rgsproc task, and only the first-order spectra of the two RGS detectors were analyzed. The spectra were rebinned by a factor of 16 .

In order to have information about the flux of the three sources in the optical and ultraviolet, we also analyzed the Optical/UV Monitor telescope (OM, Mason et al. 2001) data with the task omichain. 


\subsection{Swift XRT, BAT and UVOT}

Two pointed Swift observations of ESO 140-43 were also analyzed. Swift (Gehrels et al. 2004) carries two narrow-field instruments, XRT (Burrows et al. 2005) with an energy range $0.3-10.0 \mathrm{keV}$ and UVOT (Roming et al. 2005), which is equipped with six filters in the optical and UV range. The XRT and UVOT data were analyzed with HEADAS version 6.6.1, and applying the latest calibration files as available in January 2009. The analysis of XRT data was performed with the extraction of the source and the background spectra from the event lists using circular regions, in a similar way to what was done for the EPIC analysis. The ancillary response matrices were generated with the task xrtmkarf. The spectra were grouped to have at least 20 counts per bin.

The Seyfert galaxies ESO 140-43 and UGC 3142 were detected by Swift/BAT in the 22 month survey (Tueller et al. 2010) in the $14-195 \mathrm{keV}$ band with a significance of $4.6 \sigma$ and $4.9 \sigma$, respectively. The spectra used in this work are provided by the BAT team ${ }^{1}$. The Seyfert 2 ESO 383-18 was detected with a significance of $8.6 \sigma$ by BAT within the 39 month survey presented by Cusumano et al. (2010).

The UVOT analysis was performed creating a source and a background region file, and then using the task uvotsource to extract the flux values.

\subsection{INTEGRAL IBIS/ISGRI}

For the analysis of the IBIS/ISGRI spectra we used the observations for which the sources were at less than $10^{\circ}$ from the instrument axis. Note that because of the nature of coded mask imaging the whole sky image taken by the instrument has to be considered in the analysis, because all sources in the field of view contribute to the background (Caroli et al. 1987). The exposures in Table 2 are the ISGRI effective on-source times. The analysis was performed using version 7.0 of the Offline Science Analysis (OSA) software distributed by the ISDC (Courvoisier et al. 2003). The analysis of the IBIS/ISGRI data is based on a cross-correlation procedure between the recorded image on the detector plane and a decoding array derived from the mask pattern. The ISGRI spectra were extracted with the standard method as described in Goldwurm et al. (2003): fluxes and count spectra are extracted at the source positions for each pointing in predefined energy bins. Spectra of the same source collected in different pointings are summed to obtain an averaged spectrum during the observation.

Because the full width at half maximum ( $F W H M)$ angular resolution of IBIS/ISGRI is $12 \operatorname{arcmin}$ we investigated for possible contamination from sources close to the three AGN with Simbad ${ }^{2}$ and $N E D^{3}$ and did not find any significant contamination.

\section{X-ray spectral analysis}

The spectra derived from INTEGRAL, XMM-Newton and Swift data were analyzed using XSPEC version 11.3.2 (Arnaud 1996). Throughout the paper we give symmetric and asymmetric errors which represent $1 \sigma$ and $3 \sigma$ confidence intervals, respectively.

\footnotetext{
${ }^{1}$ http://swift.gsfc.nasa.gov/docs/swift/results/ bs22mon/

2 http://simbad.u-strasbg.fr/

3 http://nedwww.ipac.caltech.edu/
}

The probabilities that additive parameters improve a fit are calculated with the F-test. The errors on the fluxes, luminosities and the equivalent widths were calculated at the $3 \sigma$ level, with 1000 Monte Carlo simulations. We added a multiplicative factor to the models in all cases, to account for cross-calibration between the two EPIC detectors. We fixed the factor to 1 for EPIC/PN and left the factor for the two MOS detectors free to vary. The value of this factor turned out, as expected, to be close to 1 within a few percent (the differences in normalization of the two detectors should be below $5-10 \%)^{4}$.

The energy of the lines was calculated in the rest frame of the host galaxies, including in the model their redshifts.

\subsection{UGC 3142}

The $0.3-10 \mathrm{keV}$ EPIC/PN and MOS spectrum of UGC 3142 cannot be well represented with a simple power law model absorbed by cold matter, resulting in $\chi^{2}=1488$ for 1028 degrees of freedom (d.o.f.). The absorbed power law gives a very flat photon index $(\Gamma \simeq 0.9)$ and results in a strong excess (Fig. 2$)$ in soft $\mathrm{X}$-ray $(E \lesssim 1 \mathrm{keV})$. In order to account for the excess we tested several models, taking into account different emission processes. Considering a thermal origin of the excess, we added an accretion disk model consisting of multiple blackbody components (implemented as diskbb in XSPEC) to the power law. Such a model does not yield a good fit $\left(\chi^{2}=1225\right.$ for 1026 d.o.f.), and the residuals still show a strong excess below $1 \mathrm{keV}$. We then modeled the excess with a second Comptonization region, using the compTT model of Titarchuk (1994). This second Comptonization region could be identified as a hot disk surface layer. Adding this model to the power law and considering absorption yields a good fit ( $\chi^{2}=1035$ for 1024 d.o.f.), but with an unlikely very steep photon index of $\Gamma=6.4_{-1.9}^{+1.4}$, and an overly strong Compton component. As an additional emission component cannot model the soft excess, we tested whether absorption could explain the observed excess. We used a multiplicative component for the absorption to account for a neutral absorber partially covering the X-ray source (implemented as pcfabs in XSPEC):

$M(E)=f \mathrm{e}^{-N_{\mathrm{H}} \sigma(E)}+(1-f)$,

where the parameter $f$ is the covering fraction, which varies between 0 (absorber out of the line of sight) and 1 (absorber completely covering the source).

Using a power law model absorbed by Galactic cold matter plus a layer of partially absorbing neutral material significantly improved the results at the $99.9 \%$ confidence level $\left(\chi^{2}=1080\right.$ for 1027 d.o.f.), and steepened the power law $\left(\Gamma=1.13_{-0.05}^{+0.04}\right)$. This power law is still quite flat, and the model does not connect with the hard X-ray spectrum obtained by INTEGRAL IBIS/ISGRI and Swift/BAT. Adding another layer of partially absorbing neutral material again the fit improved at the $99.9 \%$ confidence level $\left(\chi^{2}=1030\right.$ for 1025 d.o.f.), and resulted in a photon index of $\Gamma=1.4_{-0.1}^{+0.1}$. The two partially covering absorbers present different characteristics: one of them covers $92 \%$ of the X-ray emitting source and has a hydrogen column density of $N_{\mathrm{H}}=1.4_{-0.2}^{+0.2} \times 10^{22} \mathrm{~cm}^{-2}$, while the other has a smaller covering factor $(\simeq 57 \%)$ and a higher column density $\left(N_{\mathrm{H}}=\right.$ $\left.5_{-1}^{+1} \times 10^{22} \mathrm{~cm}^{-2}\right)$.

Although the model fits the data well, an excess is still present around $6.4 \mathrm{keV}$. Adding a Gaussian line improves the

\footnotetext{
4 http://xmm2.esac.esa.int/docs/documents/

CAL-TN-0052-5-0. eps.gz
} 


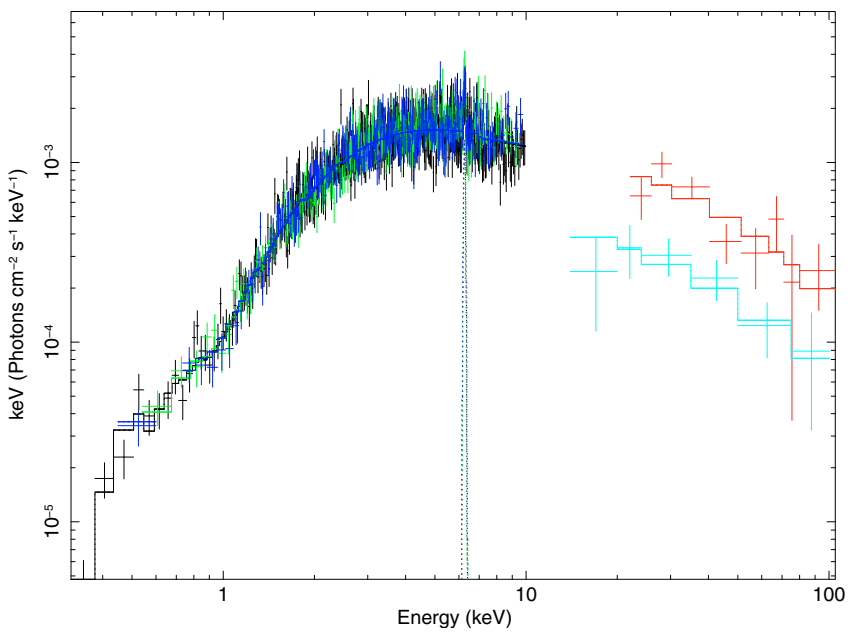

Fig. 1. $0.3-110 \mathrm{keV}$ spectrum of UGC 3142 obtained using non simultaneous EPIC (in the $0.3-10 \mathrm{keV}$ range), BAT (lower flux) and ISGRI (higher flux) data. The fit is a power law absorbed by cold Galactic matter and by two clumpy neutral absorbers, plus a Gaussian iron $\mathrm{K} \alpha$ line (last row of Table 4).

fit at the $99.9 \%$ confidence level ( $\chi^{2}=994$ for 1022 d.o.f.). The line is located at $6.38_{-0.03}^{+0.03} \mathrm{keV}$, and is therefore consistent with the iron $\mathrm{K} \alpha$ line, and has a width of $\sigma=41_{-29}^{+32} \mathrm{eV}$, and an equivalent width of $E W=83_{-60}^{+189} \mathrm{eV}$. The X-ray spectra of AGN can show an additive broad line at $6.7 \mathrm{keV}$ due to ionized iron (e.g., Matt et al. 2001). We investigated this feature adding another Gaussian line to our best model, and fixing the energy of the line at $6.7 \mathrm{keV}$, but this did not significantly improve the result $\left(\Delta \chi^{2} \simeq 0\right)$.

The RGS spectrum of UGC 3142 has a very low signal to noise ratio, and was not analyzed.

In order to search for a cut-off or a reflection hump in hard $\mathrm{X}$-ray, we combined the EPIC data with the Swift/BAT and INTEGRAL IBIS/ISGRI spectra. The $0.3-110 \mathrm{keV}$ spectrum is well represented ( $\chi^{2}=1011$ for 1034 d.o.f.) by the best model for the EPIC data alone, as presented above (power law absorbed by two layers of partially covering material). Adding a highenergy cut-off does not significantly improve the fit $\left(\chi^{2}=1008\right.$ for 1033 d.o.f.). The high-energy cut-off is nevertheless constrained $\left(E_{\mathrm{C}}=63_{-23}^{+118} \mathrm{keV}\right)$, but higher quality hard X-ray data will be necessary to verify it. As the observations were not simultaneous we added a cross-calibration constant to the model, which resulted to be $C_{\mathrm{I}}=1.4$ and $C_{\mathrm{B}}=0.5$, for ISGRI and BAT, respectively. We also tested the presence of a Compton reflection hump using the pexrav model in XSPEC, which implies the presence of reflection due to neutral material. We fixed the inclination angle at $i=30^{\circ}$ (as a common assumption for type 1 AGN, see e.g. Matt et al. 2006; Decarli et al. 2008), and found that the data are consistent with such a feature, although the reflection normalization $R$ and the cut-off energy $E_{\mathrm{C}}$ are not constrained, and have a strong degeneracy. The value of $R$ in particular is consistent with no reflection $\left(R=1_{-1}^{+2}\right)$.

We can thus conclude that the best model for the 0.3-110 keV spectrum of UGC 3142 is a power law absorbed by two layers of partially covering material plus a Gaussian line. The results of the spectral analysis are reported in Table 4.

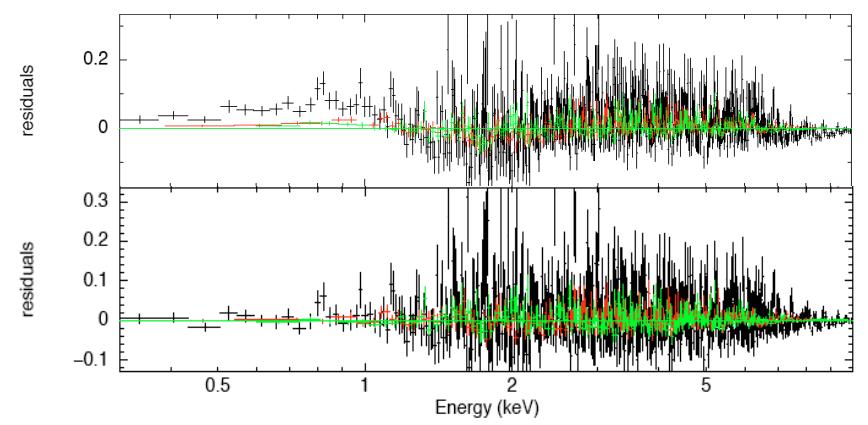

Fig. 2. UGC 3142 EPIC/PN (in black) and MOS residuals obtained with the simple power law model (upper panel) and the best (lower panel) model, respectively, as described in the first and third row of Table 4.

\subsection{ESO $383-18$}

Applying a simple power law absorbed by cold matter into the EPIC spectrum $(0.3-10 \mathrm{keV})$ of ESO $383-18$ yields a poor fit $\left(\chi^{2}=1087\right.$ for 432 d.o.f. $)$, with a low photon index $\Gamma=1.2 \pm$ 0.1 . As in the case of UGC 3142, the spectrum of ESO 383-18 presents an excess in the soft X-rays and one around $6.4 \mathrm{keV}$ (Fig. 4), when a simple power law model is applied.

Adding to the power law continuum a thermal or a nonthermal component does not represent the emission at low energies $\left(\chi^{2}=930\right.$ for 430 d.o.f. and $\chi^{2}=1051$ for 429 d.o.f., for the diskbb and comptt model, respectively). Applying a power law model absorbed by Galactic cold matter plus a layer of partially absorbing neutral material improves the results at the $99.9 \%$ confidence level ( $\chi^{2}=533$ for 431 d.o.f.), and steepens the power law $\left(\Gamma=1.47_{-0.05}^{+0.03}\right)$. Adding another layer of partially absorbing neutral material again improves the result at the $99.9 \%$ confidence level $\left(\chi^{2}=504\right.$ for 429 d.o.f.) and gives a photon index of $\Gamma=1.8_{-0.1}^{+0.1}$. One of the absorbers almost completely covers the X-ray source $\left(f=0.97_{-0.04}^{+0.02}\right)$ and has a column density of $N_{\mathrm{H}}=10_{-2}^{+1} \times 10^{22} \mathrm{~cm}^{-2}$, while the other one has $f=0.86_{-0.05}^{+0.05}$ and a higher column density $\left(N_{\mathrm{H}}=20_{-3}^{+2} \times 10^{22} \mathrm{~cm}^{-2}\right)$. To this model we added a Gaussian line, which improves the fit again at the $99.9 \%$ confidence level ( $\chi^{2}=485$ for 426 d.o.f.). This line is consistent with the iron $\mathrm{K} \alpha$ line $\left(E=6.3_{-0.1}^{+0.1} \mathrm{keV}\right)$ and has a width of $\sigma=23_{-32}^{+100} \mathrm{eV}$, and an equivalent width of $E W=76_{-66}^{+93} \mathrm{eV}$. As for UGC 3142, adding another Gaussian component at $6.7 \mathrm{keV}$ to the neutral iron line does not significantly improve the fit $\left(\Delta \chi^{2} \simeq 0\right)$. Although the two absorbers have values of column density and covering fraction within the $3 \sigma$ range of each other, the second absorber has been proven to be statistically required.

This model still displays an excess between $0.6-0.7 \mathrm{keV}$ in both PN and MOS data. This excess could be due to the OVIII Ly $\alpha$ emission line (at $0.65 \mathrm{keV}$ in the rest frame of the source). Implementing this feature in the model as a Gaussian line $\left(E=0.62_{-0.02}^{+0.02} \mathrm{keV}\right)$ improves the result at the $99 \%$ confidence level ( $\chi^{2}=461$ for 423 d.o.f.), but the data are not sufficient to significantly constrain the equivalent width of the line: $E W=144_{-137}^{+200} \mathrm{eV}$.

Due to its very low signal-to-noise ratio the RGS spectrum of ESO 383-18 was not analyzed.

The Seyfert galaxy ESO 383-18 was not detected within the 22-months BAT survey, and the INTEGRAL IBIS/ISGRI spectrum of ESO $383-18$ in the $20-100 \mathrm{keV}$ emission is well represented by a simple power law with a photon index of $\Gamma=$ $1.9_{-0.3}^{+0.4}$, consistent with the value obtained from the EPIC spectra. Combining the two spectra and using the best EPIC model 
Table 4. Results obtained from the X-ray spectral analysis of UGC 3142.

\begin{tabular}{|c|c|c|c|c|c|c|c|c|}
\hline Instruments & $\begin{array}{l}\text { Energy } \\
{[\mathrm{keV}]}\end{array}$ & $\chi^{2}$ (d.o.f.) & $\Gamma$ & $\begin{array}{l}N_{\mathrm{H}} \\
{\left[10^{20} \mathrm{~cm}^{-2}\right]}\end{array}$ & $\begin{array}{l}N_{\mathrm{H}}^{1} \\
{\left[10^{22} \mathrm{~cm}^{-2}\right]}\end{array}$ & $f^{1}$ & $\begin{array}{l}N_{\mathrm{H}}^{2} \\
{\left[10^{22} \mathrm{~cm}^{-2}\right]}\end{array}$ & $f^{2}$ \\
\hline $\mathrm{EPIC}^{a}$ & $0.3-10$ & $1488(1028)$ & $0.9_{-0.1}^{+0.1}$ & $1.4_{-0.1}^{+0.1}$ & - & - & - & - \\
\hline $\mathrm{EPIC}^{b}$ & $0.3-10$ & 1080 (1027) & $1.13_{-0.05}^{+0.04}$ & 0.19 & $2.1_{-0.1}^{+0.1}$ & $0.92_{-0.01}^{+0.01}$ & - & - \\
\hline $\mathrm{EPIC}^{c}$ & $0.3-10$ & $994(1022)$ & $1.4_{-0.1}^{+0.103}$ & 0.19 & $1.4_{-0.2}^{+0.1}$ & $0.92_{-0.02}^{+0.01}$ & $5_{-1}^{+1}$ & $0.57_{-0.09}^{+0.08}$ \\
\hline$(\mathrm{EPIC}+\mathrm{ISGRI}+\mathrm{BAT})^{c}$ & $0.3-110$ & 1011 (1034) & $1.5_{-0.1}^{+0.1}$ & 0.19 & $1.4_{-0.4}^{+0.3}$ & $0.92_{-0.06}^{+0.02}$ & $4_{-1}^{+2}$ & $0.6_{-0.1}^{+0.2}$ \\
\hline
\end{tabular}

Notes. The data were modeled with an absorbed power law model $(a)$, a power law absorbed by Galactic cold matter plus a layer of partially absorbing neutral material $(b)$, and a power law absorbed by Galactic cold matter and two layers of partially absorbing neutral material, plus a Gaussian $\mathrm{K} \alpha$ iron line (c). EPIC includes PN, MOS1 and MOS2 data.

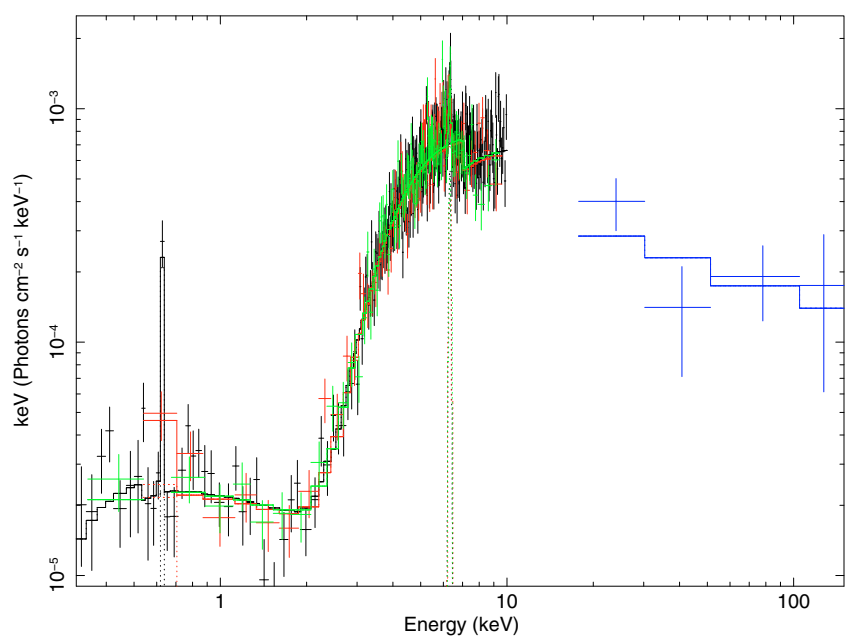

Fig. 3. 0.3-110 keV spectrum of ESO 383-18 obtained with non simultaneous EPIC (in the $0.3-10 \mathrm{keV}$ range) and ISGRI data. The fit is a power law absorbed by cold Galactic matter and by two clumpy neutral absorbers plus two Gaussian emission lines (last row of Table 5).

we obtain parameters consistent with those obtained previously (Table 5), due to the much richer statistics of EPIC. Due to the low quality of the IBIS/ISGRI data, it is not possible to constrain the presence of a cut-off, or of a reflection component $(R \simeq 0)$ in the spectrum using pexrav. The fit included an inter-calibration constant $\left(C_{\mathrm{I}}=0.7\right)$ to account for variability, because ISGRI and EPIC observations were not simultaneous, and the inclination angle was set to $i=60^{\circ}$ (Kinney et al. 2000) because this source is a type $2 \mathrm{AGN}$.

We can conclude that the best model for the spectrum of ESO 383-18 is a power law absorbed by cold Galactic matter and by a double layer of partially covering material, plus two Gaussian lines.

\subsection{ESO $140-43$}

Two XMM-Newton observations of ESO 140-43 were performed, the first in September 2005 and the second in March 2006.

As in for ESO 383-18 and UGC 3142, in the $0.3-10 \mathrm{keV}$ energy range a simple power law absorbed by cold neutral matter does not provide a good representation of the EPIC/PN and MOS data for both observations $\left(\chi^{2}=18189\right.$ for 2153 d.o.f. and $\chi^{2}=3234$ for 947 d.o.f., for the 2005 and 2006 observation, respectively). It also results in a flat power law with a strong soft excess (Figs. 8 and 10). We used again a more complex cold absorber, but in this case the data are not consistent with neutral

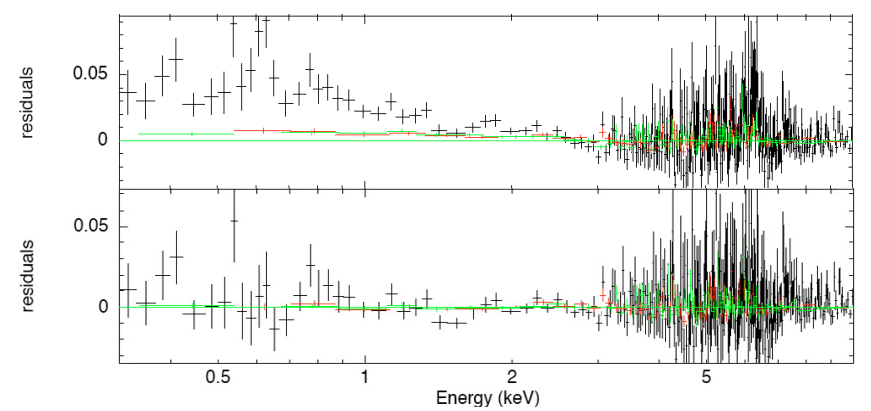

Fig. 4. ESO 383-18 EPIC/PN (in black) and MOS residuals obtained with the worst (upper panel) and best (lower panel) model, respectively the first and third row of Table 5.

absorption. Adopting a clumpy absorber model results in a chisquared of $\chi^{2}=10861$ for 2151 d.o.f. for the EPIC spectrum of the 2005-observation. Jiménez-Bailón et al. (2008) found evidence of absorption features produced by highly ionized material in the RGS spectrum of ESO 140-43, due to three warm absorbers in the line of sight.

We analyzed the characteristics of the ionized matter with a model that uses the XSTAR code (e.g., Kallman et al. 1996; Kallman \& Bautista 2001). This calculates the physical conditions and absorption-emission spectra of photo-ionized gases, and considers variable abundances. We used zxipcf, a model of partial covering absorption by partially ionized material, which uses a grid of XSTAR photo-ionized absorption models. In this model the state of the warm absorber is characterized by the column density of the absorber $N_{\mathrm{H}}$, its covering fraction $f$, and the logarithm of the ionization parameter $\xi$, defined as

$\xi=\frac{L_{\mathrm{ion}}}{n r^{2}}$

where $L_{\text {ion }}$ is the isotropic luminosity of the ionizing source in the $5 \mathrm{eV}-300 \mathrm{keV}$ energy range, $r$ is the distance of the warm plasma to the source of radiation, and $n$ is the proton density. This model is the ionized equivalent of the pcfabs model used for UGC 3142 and ESO 383-18.

In the case of the 2005 EPIC observation of ESO 140-43 adding a clumpy warm absorber improves the model $\left(\chi^{2}=3409\right.$ for 2151 d.o.f.) with respect to a simple power law absorbed by cold matter. A second clumpy warm absorber again improves the result at a $99.9 \%$ confidence level $\left(\chi^{2}=2800\right.$ for 2148 d.o.f. $)$. The best model is obtained adding a third clumpy warm absorber, which improves the fit again at the $99.9 \%$ confidence level $\left(\chi^{2}=2575\right.$ for 2145 d.o.f.). The best model has a photon index of $\Gamma=1.89_{-0.01}^{+0.01}$ and three warm absorbers with quite different characteristics. The low ionized absorber (hereafter LIA) 
Table 5. Results obtained from the X-ray spectral analysis of ESO 383-18.

\begin{tabular}{|c|c|c|c|c|c|c|c|c|}
\hline Instruments & $\begin{array}{l}\text { Energy } \\
{[\mathrm{keV}]}\end{array}$ & $\chi^{2}$ (d.o.f.) & $\Gamma$ & $\begin{array}{l}N_{\mathrm{H}} \\
{\left[10^{20} \mathrm{~cm}^{-2}\right]}\end{array}$ & $\begin{array}{l}N_{\mathrm{H}}^{1} \\
{\left[10^{22} \mathrm{~cm}^{-2}\right]}\end{array}$ & $f^{1}$ & $\begin{array}{l}N_{\mathrm{H}}^{2} \\
{\left[10^{22} \mathrm{~cm}^{-2}\right]}\end{array}$ & $f^{2}$ \\
\hline $\mathrm{EPIC}^{a}$ & $0.3-10$ & 1087 (432) & $1.2 \pm 0.1$ & $15 \pm 1$ & - & - & - & - \\
\hline $\mathrm{EPIC}^{b}$ & $0.3-10$ & $533(431)$ & $1.47_{-0.05}^{+0.03}$ & 0.042 & $19.5_{-07}^{+0.7}$ & $0.989_{-0.003}^{+0.002}$ & - & - \\
\hline EPIC $^{c}$ & $0.3-10$ & $461(423)$ & $1.8_{-0.1}^{+0.1}$ & 0.042 & $10_{-2}^{+1}$ & $0.97_{-0.04}^{+0.02}$ & $20_{-3}^{+2}$ & $0.86_{-0.05}^{+0.05}$ \\
\hline$(\mathrm{EPIC}+\mathrm{ISGRI})^{c}$ & $0.3-110$ & $472(426)$ & $1.7_{-0.2}^{+0.2}$ & 0.042 & $11_{-5}^{+17}$ & $0.95_{-0.15}^{+0.03}$ & $15_{-7}^{+4}$ & $0.85_{-0.07}^{+0.06}$ \\
\hline
\end{tabular}

Notes. The data were modeled with an absorbed power law model $(a)$, a power law absorbed by Galactic cold matter plus a layer of partially absorbing neutral material $(b)$, and a power law plus two Gaussian lines (representing the iron K $\alpha$ and the OVIII Ly $\alpha$ emission lines)s absorbed by Galactic cold matter and by two layers of partially absorbing neutral material (c). EPIC includes PN, MOS1 and MOS2 data.

$\left(\log \xi=0.28_{-0.03}^{+0.05} \mathrm{erg}^{-1} \mathrm{~cm} \mathrm{~s}^{-1}\right)$ with a hydrogen column density of $N_{\mathrm{H}}^{\mathrm{L}}=3.7_{-0.7}^{+0.3} \times 10^{21} \mathrm{~cm}^{-2}$ covers a fraction of $f=0.70_{-0.04}^{+0.04}$ of the X-ray source. The intermediate ionized absorber (IIA) $\left(\log \xi=1.94_{-0.04}^{+0.02} \mathrm{erg} \mathrm{cm} \mathrm{s}^{-1}\right)$ has a higher hydrogen column density of $N_{\mathrm{H}}^{\mathrm{I}}=2.99_{-0.01}^{+0.07} \times 10^{22} \mathrm{~cm}^{-2}$ and a covering factor of $f=0.98_{-0.05}^{+0.01}$. The third absorber has a value of the ionization parameter higher than the other two $\left(\log \xi=2.73_{-0.04}^{+0.06} \mathrm{erg} \mathrm{cm} \mathrm{s}^{-1}\right)$, and will therefore be called high ionization absorber (HIA). Its hydrogen column density and covering factor are $N_{\mathrm{H}}^{\mathrm{H}}=1.1_{-0.2}^{+0.2} \times$ $10^{22} \mathrm{~cm}^{-2}$ and $f=0.99_{-0.01}^{+0.01}$, consistent with full coverage. The residuals of this fit (Fig. 8) show that there are still some components in the spectrum which are not yet accounted for, although the model provides an acceptable fit $\left(\chi_{v}^{2}=1.17\right)$. Higher quality data would be needed to investigate whether another absorption component could explain the remaining residuals.

The EPIC/PN and MOS X-ray spectra of the 2006 XMM-Newton observation of ESO 140-43 were analyzed with the models reported above. As for the 2005 observation the best fit $\left(\chi^{2}=1063\right.$ for 940 d.o.f.) is obtained with a power law absorbed by three layers of partially covering ionized matter. The value of the photon index obtained $\left(\Gamma=1.9_{-0.2}^{+0.2}\right)$ is consistent with that of the 2005 observation, while the parameters of the absorbers show several important differences. The LIA has an even lower ionization parameter $\left(\log \xi^{\mathrm{L}}=-1.3_{-0.2}^{+1.0} \mathrm{erg} \mathrm{cm} \mathrm{s}^{-1}\right)$ than that of the 2005 observation, it has a higher hydrogen column density $\left(N_{\mathrm{H}}^{\mathrm{L}}=1.6_{-0.8}^{+0.5} \times 10^{22} \mathrm{~cm}^{-2}\right)$ and covers a bigger fraction of the X-ray source $\left(f=0.88_{-0.09}^{+0.03}\right)$. The IIA presents a value of the ionization parameter $\left(\log \xi^{\mathrm{I}}=1.89_{-0.4}^{+0.03} \mathrm{erg} \mathrm{cm} \mathrm{s}^{-1}\right)$ and of the covering factor $\left(f=0.86_{-0.04}^{+0.09}\right)$ consistent with the $2005 \mathrm{ob}-$ servation, while its column density $\left(N_{\mathrm{H}}^{\mathrm{I}}=11_{-6}^{+3} \times 10^{22} \mathrm{~cm}^{-2}\right)$ is remarkably higher. The HIA has a higher value of the ionization parameter $\left(\log \xi^{\mathrm{H}}=3.6_{-0.2}^{+0.8} \mathrm{erg} \mathrm{cm} \mathrm{s}^{-1}\right)$ and of the column density $\left(N_{\mathrm{H}}^{\mathrm{H}}=8_{-6}^{+12} \times 10^{22} \mathrm{~cm}^{-2}\right)$ with respect to the 2005 observation, while its covering factor $\left(f=0.86_{-0.04}^{+0.09}\right)$ is consistent.

The spectra of the two observations are reported together in Fig. 5.

A layer of neutral matter representing the Galactic absorption $\left(N_{\mathrm{H}}^{\mathrm{Gal}}=7.34 \times 10^{20} \mathrm{~cm}^{-2}\right)$ was added to all the models reported above. To discard neutral absorbers we added a layer of cold material to the models and found that its hydrogen column density is consistent with 0 for both observations, thus it can be ignored.

The first evidence of a $\mathrm{K} \alpha$ iron line in the X-ray spectrum of ESO 140-43 was found through the analysis of EXOSAT data. Ghosh \& Soundararajaperumal (1992) detected an excess around $6 \mathrm{keV}$, which they interpreted as an iron line of the equivalent width $E W=354 \pm 144 \mathrm{eV}$. We added a Gaussian line to

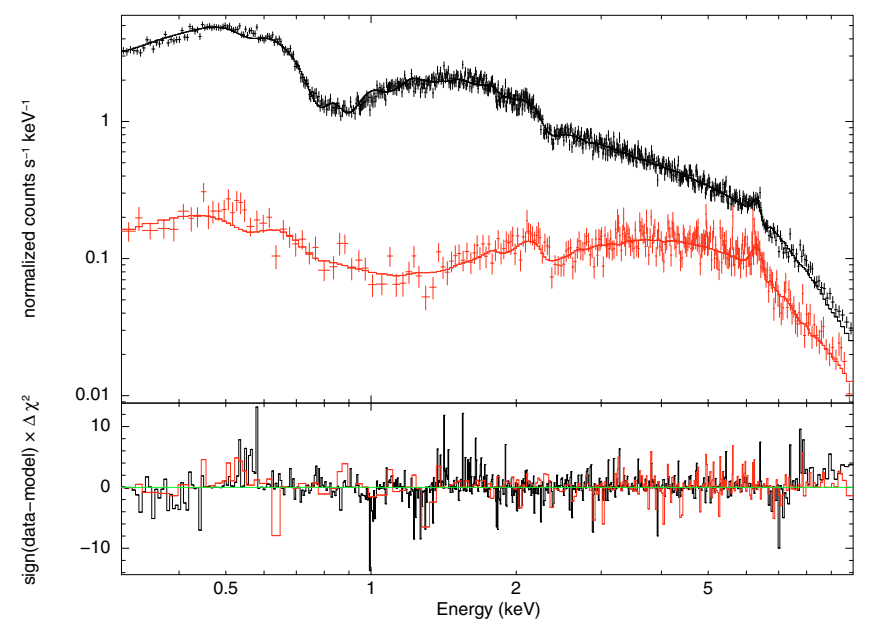

Fig. 5. EPIC/PN spectra of the 2005 (in black) and 2006 observation of ESO 140-43. The continuous lines represent the best models to the respective data-sets, as reported in Table 6 .

the best-fit model and found that this component improves the fit at the $99.9 \%$ confidence level for both observations $\left(\chi^{2}=2511\right.$ for 2142 d.o.f. and $\chi^{2}=2012$ for 937 d.o.f., for the 2005 and 2006 observation, respectively). From the spectrum of the 2005observation we obtained a line located at $6.40_{-0.03}^{+0.03} \mathrm{keV}$, with a width of $\sigma=83_{-32}^{+34} \mathrm{eV}$, an equivalent width of $E W=118_{-53}^{+51} \mathrm{eV}$ and a flux of $F_{\mathrm{K} \alpha}=4.4_{-0.9}^{+0.9} \times 10^{-15} \mathrm{erg} \mathrm{cm}^{-2} \mathrm{~s}^{-1}$. In the 2006observation spectrum the $\mathrm{K} \alpha$ iron line is at $6.39_{-0.05}^{+0.05} \mathrm{keV}$, has a width of $\sigma=112_{-37}^{+57} \mathrm{eV}$, an equivalent width of $E W=$ $161_{-71}^{+10} \mathrm{eV}$ ) and a flux of $F_{\mathrm{K} \alpha}=2.4_{-0.6}^{+0.7} \times 10^{-15} \mathrm{erg} \mathrm{cm}^{-2} \mathrm{~s}^{-1}$. This line is for both observations consistent with the $\mathrm{K} \alpha$ iron line. For both observations adding another Gaussian line at $6.7 \mathrm{kev}$ does not significantly improve the fit $\left(\Delta \chi^{2} \simeq 0\right)$, which allows us to exclude an additive broad component to the neutral iron line. The results obtained with this model are reported in Table 6.

While the RGS spectrum of the 2005 observation (Fig. 6) shows a number of interesting features in the $0.3-2 \mathrm{keV}$ energy range, the one obtained during the 2006 observation has a very low signal to noise ratio. The most evident absorption features detected by RGS during the 2005-observation are (the energy are given in the rest frame of the AGN): the N VII Ly $\alpha$ $(0.5 \mathrm{keV} ; 24.8 \AA)$, the O VIII Ly $\alpha(0.65 \mathrm{keV} ; 19 \AA)$ and possibly the $\mathrm{Ne} \mathrm{X} \mathrm{Ly} \alpha(1.02 \mathrm{keV} ; 12.1 \AA)$ line. In Fig. 6 we plot the RGS spectrum using the best EPIC model, which fits the continuum well, but not the OVIII Ly $\alpha$ line and the continuum next to it, similarly to what happens in the best fit of Jiménez-Bailón et al. (2008). A more detailed analysis with 
Table 6. Results obtained from the X-ray spectral analysis of the two XMM-Newton EPIC and the two Swift/XRT observations of ESO 140-43.

\begin{tabular}{|c|c|c|c|c|c|c|c|c|c|c|}
\hline$\chi^{2}$ (d.o.f.) & $\Gamma$ & $\begin{array}{l}N_{\mathrm{H}}^{1} \\
{\left[10^{22} \mathrm{~cm}^{-2}\right]}\end{array}$ & $\begin{array}{l}\log \xi^{1} \\
{\left[\mathrm{erg} \mathrm{cm} \mathrm{s}^{-1}\right]}\end{array}$ & $f^{1}$ & $\begin{array}{l}N_{\mathrm{H}}^{2} \\
{\left[10^{22} \mathrm{~cm}^{-2}\right]}\end{array}$ & $\begin{array}{l}\log \xi^{2} \\
{\left[\mathrm{ergcm} \mathrm{s}^{-1}\right]}\end{array}$ & $f^{2}$ & $\begin{array}{l}N_{\mathrm{H}}^{3} \\
{\left[10^{22} \mathrm{~cm}^{-2}\right]}\end{array}$ & $\begin{array}{l}\log \xi^{3} \\
{\left[\mathrm{erg} \mathrm{cm} \mathrm{s}^{-1}\right]}\end{array}$ & $f^{3}$ \\
\hline \multicolumn{11}{|c|}{ XMM-Newton EPIC/PN and MOS } \\
\hline & & & LIA & & & IIA & & & HIA & \\
\hline \multicolumn{11}{|c|}{2005 Observation } \\
\hline $2511(2142)$ & $1.89_{-0.01}^{+0.01}$ & $0.37_{-0.07}^{+0.03}$ & $0.28_{-0.03}^{+0.05}$ & $0.70_{-0.04}^{+0.04}$ & $2.99_{-0.01}^{+0.07}$ & $1.94_{-0.04}^{+0.02}$ & $0.98_{-0.05}^{+0.01}$ & $1.1_{-0.2}^{+0.2}$ & $2.73_{-0.04}^{+0.06}$ & $0.99_{-0.01}^{+0.01}$ \\
\hline \multicolumn{11}{|c|}{2006 Observation } \\
\hline 1012(937) & $1.9_{-0.2}^{+0.2}$ & $1.6_{-0.8}^{+0.5}$ & $-1.3_{-0.2}^{+1.0}$ & $0.88_{-0.09}^{+0.03}$ & $11_{-6}^{+3}$ & $1.89_{-0.4}^{+0.03}$ & $0.86_{-0.04}^{+0.09}$ & $8_{-6}^{+12}$ & $3.6_{-0.2}^{+0.8}$ & $0.99_{-0.01}^{+0.01}$ \\
\hline \multicolumn{11}{|l|}{ Swift/XRT } \\
\hline \multicolumn{11}{|c|}{ First observation } \\
\hline $13.1(13)$ & $3.8_{-2.2}^{+1.0}$ & $7_{-6}^{+13}$ & $-0.5_{-0.7}^{+2.3}$ & $0.99_{-0.01}^{+0.01}$ & & & & & & \\
\hline \multicolumn{11}{|c|}{ Second observation } \\
\hline $12.9(12)$ & $2.9_{-1.2}^{+1.4}$ & $5_{-1}^{+2}$ & $-0.5_{-0.1}^{+2.3}$ & $0.996_{-0.03}^{+0.004}$ & & & & & & \\
\hline
\end{tabular}

Notes. The model used is a power law plus a iron $\mathrm{K} \alpha$ line absorbed by three layers of partially covering ionized matter for EPIC, and a power law absorbed by one layer of partially covering ionized material for XRT. An additional layer of cold matter representing neutral Galactic absorption was used, fixing the column density at $N_{\mathrm{H}}^{\mathrm{G} a l}=7.3 \times 10^{20} \mathrm{~cm}^{-2}$.

different parameters and possibly other model components is required, but is out of the scope of this paper.

We also analyzed the less significant data obtained during the two Swift/XRT observations. The two observations were performed a few days apart, but because the flux varied, we analyzed them individually. Fitting the spectra with a simple power law we obtained $\chi^{2}=18.3$ for 15 d.o.f. and $\chi^{2}=23$ for 14 d.o.f., for the first and second observation, respectively. Due to the low quality of the data it is impossible to resolve the three absorbers, thus we applied a simpler model than that used for EPIC data: a power law absorbed by only one layer of clumpy ionized material. This model fits well both observations and the results are reported in Table 6.

Analyzing IBIS/ISGRI and BAT spectra together with the most significant EPIC spectra, we checked for a cut-off and/or reflection from neutral material. No evidence of an exponential cut-off was found in the data, the energy of the cut-off reaches indeed the upper limit allowed by XSPEC and is not constrained $\left(E_{\mathrm{C}}=500 \pm 1000 \mathrm{keV}\right)$. With the pexrav reflection model (fixing the inclination angle to $i=30^{\circ}$ and the cut-off at $E_{\mathrm{C}}=10000 \mathrm{keV}$ ) we found that the fit improves at the $98 \%$ confidence level $\left(\chi^{2}=2540\right.$ for 2150 d.o.f. and $\chi^{2}=2546$ for 2151 d.o.f., for the new and the old model, respectively). The value of the reflection obtained is $R=0.6_{-0.2}^{+0.1}$.

We can conclude that the best model for the $0.3-110 \mathrm{keV}$ spectrum of ESO 140-43 is that of a power law reflected by neutral material and absorbed by three layers of partially covering ionized material, plus a iron $\mathrm{K} \alpha$ line.

\section{Variability analysis}

\subsection{X-ray variability}

We analyzed the $0.3-10 \mathrm{keV}$ EPIC/PN light curves (with a binning of $200 \mathrm{~s}$ ) of the three Seyfert galaxies (Fig. 11) and investigated whether variations in the absorbers can account for variability. We tested the light curves for a constant flux model, and rejected the null hypothesis (i.e. flux not variable) for values of the probability $p$ lower than $5 \%$.

The light curve of UGC 3142 is consistent with a constant flux $\left(\chi^{2}=54\right.$ for 49 d.o.f., which leads to a probability of $p \sim 30 \%$ ), with a mean count-rate of $\bar{f}=2.08 \pm 0.02 \mathrm{ct} \mathrm{s}^{-1}$. The light curve of ESO 383-18 shows significant variability during

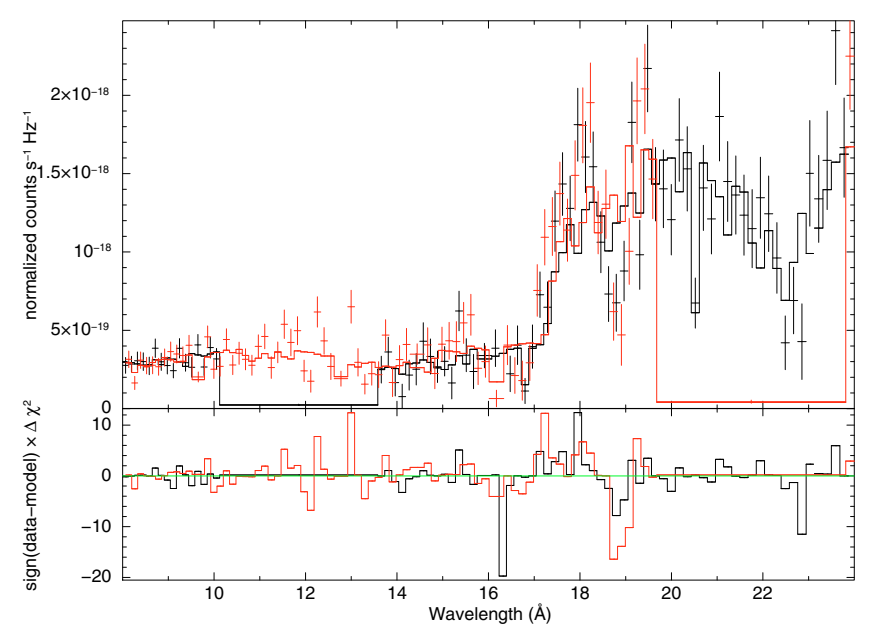

Fig. 6. Extract of the RGS spectrum of the 2005 observation of ESO 140-43, the solid line represents the best EPIC model (Table 6).

the observation, with $\chi^{2}=187.5$ for 70 d.o.f. $(p \sim 0 \%)$ for the assumption of constant flux. In order to better estimate the variability of these two light-curves we calculated their fractional rms variability amplitude $F_{\text {var }}$ (as defined in Eq. (10) in Vaughan et al. 2003). For UGC 3142 we obtained $F_{\text {var }}=1.5 \pm 2 \%$, compatible with the lack of variability found above, while for ESO 383-18 we obtained $F_{\text {var }}=14 \pm 2 \%$. The errors on $F_{\text {var }}$ were calculated with Eq. (B2) in Vaughan et al. (2003).

The light curves for the two observations of ESO 140-43 show evidence of variable flux, with $\chi^{2}=1575$ for 107 d.o.f. $(p \sim 0 \%)$ and $\chi^{2}=257$ for 99 d.o.f. $(p \sim 0 \%)$, for the 2005 and 2006 observations, respectively. The values of the fractional rms variability amplitude are consistent, $F_{\text {var }}=8.9 \pm 0.3 \%$ and $F_{\text {var }}=9 \pm 1 \%$, for the 2005 and 2006 observation, respectively.

The 2005-observation seems to be characterized by two different (at the $1 \sigma$ level) flux states. The light curve indeed shows a constant flux of about $8 \mathrm{ct} / \mathrm{s}$ for the first $10 \mathrm{ks}$, then a dip and a flux oscillating around $6.7 \mathrm{ct} / \mathrm{s}$ for about $9 \mathrm{ks}$, then increasing again up to $10 \mathrm{ct} / \mathrm{s}$. In order to test whether the different flux states can be accounted for by a change in the absorber or in the spectral shape, we divided the light curve into two parts: part 1, between 0 and $10 \mathrm{ks}$ for the high flux state, and part 2, between 10 and $19 \mathrm{ks}$, for the low flux state. These two 


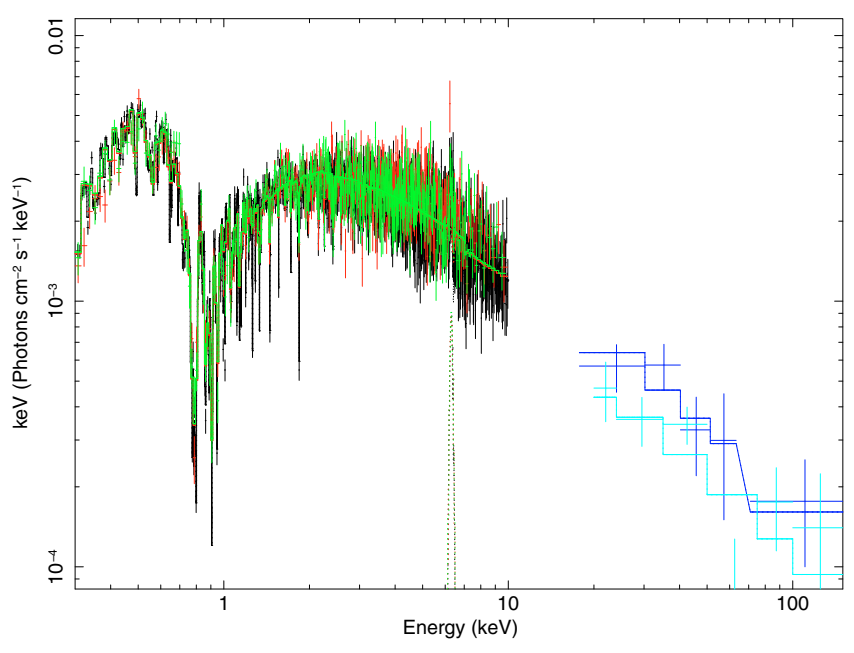

Fig. 7. $0.3-110 \mathrm{keV}$ spectrum of ESO 140-43 obtained with the 2005 $X M M$-Newton EPIC (in the 0.3-10 keV range), and non simultaneous BAT (lower flux) and IBIS/ISGRI (higher flux) data. The model used is a power law absorbed by cold Galactic matter and by three clumpy ionized absorbers, plus a iron $\mathrm{K} \alpha$ line and a reflection component.

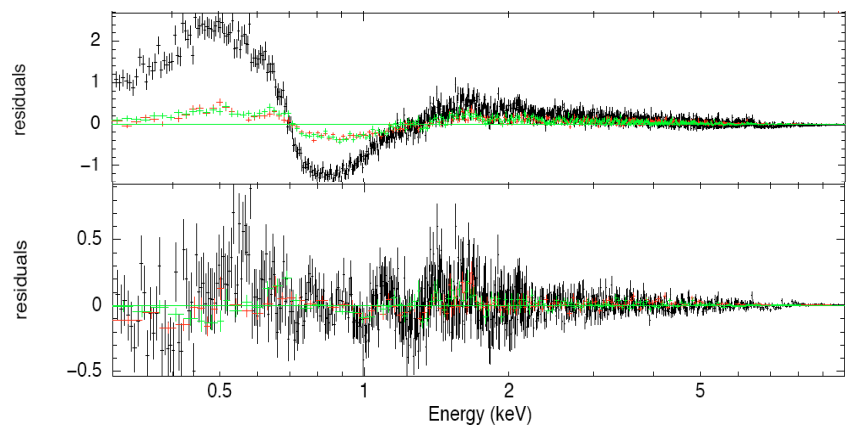

Fig. 8. EPIC/PN (in black) and MOS residuals of the 2005 $X M M$-Newton observation obtained with the worst (simple power law, top panel) and best fit (power law absorbed by cold Galactic matter and by three clumpy ionized absorbers, plus a iron $\mathrm{K} \alpha$ line, bottom panel).

light-curves have consistent intrinsic variabilities of a few percents $\left(F_{\mathrm{var}}^{1}=5.1 \pm 0.4 \%\right.$ and $\left.F_{\mathrm{var}}^{2}=5.2 \pm 0.5 \%\right)$. In order to find a possible explanation for these two flux states, we analyzed the spectra obtained from the two time intervals (Fig. 12). We fitted the two spectra first freezing all the parameters except the normalization of the power law, and then also letting the other parameters free to vary. We explored several possibilities, as variations of the column densities, of the ionization parameters or of the covering fraction. We found that the only parameter, besides the normalization, whose variation could explain the different spectra is the covering factor of the LIA. In particular a variation of this parameter (from $f_{1}^{\text {LIA }}=0.77_{-0.02}^{+0.01}$ to $f_{2}^{\text {LIA }}=0.65_{-0.03}^{+0.02}$ ) is necessary at the $99.9 \%$ confidence level to account for these spectral differences.

In Table 8 we report the fluxes of the three sources based both on this work and previous literature. A strong variation in flux in the $14-150 \mathrm{keV}$ band is evident between ISGRI and BAT observations of UGC 3142, while the values of ESO 383-18 and ESO 140-43 are consistent, and there is no evidence of hard $\mathrm{X}$-ray variability. At lower energies flux (by a factor of 3) and spectral variability on a time-scale of months was detected between the two XMM-Newton observations of ESO 140-43. The fluxes of ESO 140-43 measured by EXOSAT are consistent with those obtained by the 2005 EPIC observation, while the flux was

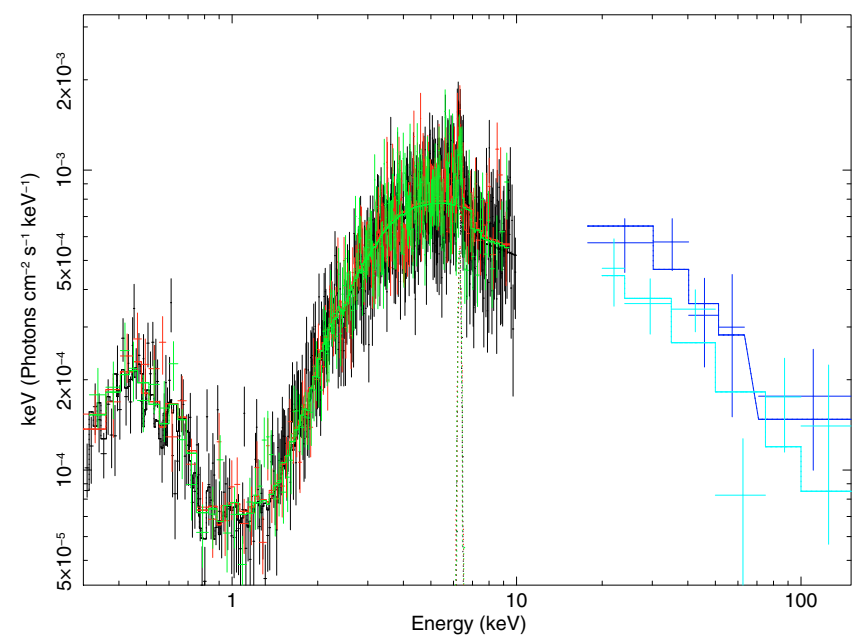

Fig. 9. 0.3-110 keV spectrum of ESO 140-43 obtained with the 2006 XMM-Newton/EPIC (in the $0.3-10 \mathrm{keV}$ range), and non simultaneous BAT (lower flux) and IBIS/ISGRI (higher flux) data. The model is a power law absorbed by cold Galactic matter and by three clumpy ionized absorbers, plus a iron $\mathrm{K} \alpha$ line and a reflection component.

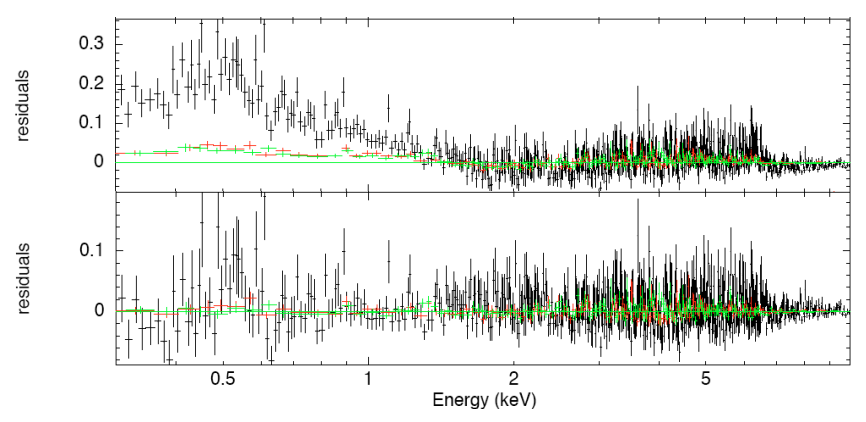

Fig. 10. EPIC/PN (in black) and MOS residuals of the 2006 $X M M$-Newton observation obtained with the worst (simple power law, top panel) and best fit (power law absorbed by cold Galactic matter and by three clumpy ionized absorbers, plus a Gaussian $\mathrm{K} \alpha$ iron line, bottom panel).

much lower during the 2006 observation. Strong variability between the ROSAT observation and the second EPIC observation was also detected.

Thus of the three Seyferts only UGC 3142 shows strong hard X-ray variability, while in soft X-ray ESO 383-18 and ESO 140-43 show both variability on the 200s timescale, and ESO 140-43 shows remarkable flux and spectral variability on a monthly timescale, as is evident from XMM-Newton and historical observations.

\subsection{Hardness ratios}

In order to probe the possible role of absorption in the 0.3-10 keV flux variability of ESO 383-18 and ESO 140-43, we used two bands, a soft $(S, 0.3-2 \mathrm{keV})$ band and a hard $(H$, $2-10 \mathrm{keV}$ ) band, and checked for variations of the hardness ratio with time. The hardness ratio is defined as

$\mathrm{HR}=\frac{H-S}{H+S}$ 
A\&A 518, A47 (2010)

Table 7. Optical and ultraviolet apparent magnitudes measured by the two different XMM-Newton/OM and Swift/XRT observations.

\begin{tabular}{|c|c|c|c|c|c|}
\hline $\begin{array}{l}\text { Instrument } \\
\text { Time }\end{array}$ & & $\begin{array}{c}\mathrm{XMM} / \mathrm{OM} \\
08-09-2005\end{array}$ & $\begin{array}{c}\mathrm{XMM} / \mathrm{OM} \\
07-03-2006\end{array}$ & $\begin{array}{c}\text { Swift/UVOT } \\
20-05-2008\end{array}$ & $\begin{array}{c}\text { Swift/UVOT } \\
25-05-2008\end{array}$ \\
\hline Band & $\begin{array}{c}\text { Wavelength } \\
\AA\end{array}$ & $\begin{array}{l}\text { Magnitude } \\
\text { mag }\end{array}$ & $\begin{array}{l}\text { Magnitude } \\
\text { mag }\end{array}$ & $\begin{array}{l}\text { Magnitude } \\
\text { mag }\end{array}$ & $\begin{array}{l}\text { Magnitude } \\
\text { mag }\end{array}$ \\
\hline $\bar{V}$ & 5430 & $14.56_{-0.01}^{+0.01}$ & $14.57_{-0.01}^{+0.01}$ & - & - \\
\hline$B$ & 4500 & $15.34_{-0.01}^{+0.01}$ & $15.37_{-0.01}^{+0.01}$ & - & - \\
\hline$U$ & 3440 & $14.90_{-0.01}^{+0.01}$ & $14.94_{-0.01}^{+0.01}$ & - & - \\
\hline$U V W 1$ & 2910 & $15.14_{-0.01}^{+0.01}$ & $15.18_{-0.01}^{+0.01}$ & - & $16.0_{-0.1}^{+0.1}$ \\
\hline UVM2 & 2310 & $16.14_{-0.04}^{+0.81}$ & $16.67_{-0.04}^{-0.81}$ & $16.8_{-0.1}^{+0.1}$ & -0.1 \\
\hline$U V W 2$ & 2120 & $16.57_{-0.08}^{+0.08}$ & - & - & - \\
\hline
\end{tabular}

Table 8. Comparison between fluxes in different energy bands obtained in this work and found in the literature.

\begin{tabular}{|c|c|c|c|c|}
\hline Instrument & $F_{0.1-2 \mathrm{keV}}$ & $F_{0.1-2.4 \mathrm{keV}}$ & $F_{2-10 \mathrm{keV}}$ & $F_{14-150 \mathrm{keV}}$ \\
\hline & {$\left[10^{-12} \mathrm{erg} \mathrm{cm}^{-2} \mathrm{~s}^{-1}\right]$} & {$\left[10^{-12} \mathrm{erg} \mathrm{cm}^{-2} \mathrm{~s}^{-1}\right]$} & {$\left[10^{-11} \mathrm{erg} \mathrm{cm}^{-2} \mathrm{~s}^{-1}\right]$} & {$\left[10^{-11} \mathrm{erg} \mathrm{cm}^{-2} \mathrm{~s}^{-1}\right]$} \\
\hline \multicolumn{5}{|l|}{ UGC 3142} \\
\hline$E P I C / \mathrm{PN}+\mathrm{MOS}$ & $0.69_{-0.11}^{+0.04}$ & $1.24_{-0.1}^{+0.1}$ & $1.7_{-02}^{+0.1}$ & - \\
\hline Swift $/ \mathrm{BAT}^{a}$ & $-\quad-0.11$ & $-\quad-0.1$ & $--^{-0.2}$ & $3.4_{-06}^{+0.6}$ \\
\hline INTEGRAL IBIS/ISGRI & - & - & - & $9.42_{-0.8}^{+0.6}$ \\
\hline \multicolumn{5}{|l|}{ ESO $383-18$} \\
\hline$E P I C / \mathrm{PN}+\mathrm{MOS}$ & $0.06_{-0.01}^{+0.18}$ & $0.08_{-0.01}^{+0.18}$ & $0.6_{-01}^{+0.1}$ & - \\
\hline Swift $/ \mathrm{BAT}^{b}$ & $-\quad-0.01$ & $-{ }^{-0.01}$ & -0.1 & $1.9_{-06}^{+0.6}$ \\
\hline INTEGRAL IBIS/ISGRI & - & - & - & $3.8_{-1.5}^{+0.8}$ \\
\hline \multicolumn{5}{|l|}{ ESO $140-43$} \\
\hline$R O S A T^{c}$ & - & $21_{-10}^{+10}$ & - & - \\
\hline$E P I C / \mathrm{PN}+\mathrm{MOS}^{1}$ & $6.5_{-0.1}^{+0.1}$ & $8.5_{-0.6}^{+10.1}$ & $2.46_{-0.04}^{+0.05}$ & - \\
\hline$E P I C / \mathrm{PN}+\mathrm{MOS}^{2}$ & $0.4_{-0.1}^{+0.1}$ & $0.5_{-0.2}^{+0.6}$ & $0.8_{-0.2}^{+0.0 .04}$ & - \\
\hline EXOSAT $^{d}$ & $8_{-3}^{+3^{-0.1}}$ & -0.2 & $2.5_{-0.2}^{-0.2}$ & - \\
\hline Swift $/ \mathrm{BAT}^{a}$ & - & - & -0.2 & $4.6_{-07}^{+0.7}$ \\
\hline INTEGRAL IBIS/ISGRI & - & - & - & $5.3_{-05}^{+0.4}$ \\
\hline
\end{tabular}

Notes. The indices 1 and 2 refer to the 2005 and 2006 XMM-Newton observation of ESO 140-43, respectively. The fluxes are model fluxes, not corrected for absorption.

${ }^{(a)}$ Tueller et al. (2010); ${ }^{(b)}$ Cusumano et al. (2010); ${ }^{(c)}$ Boller et al. (1992); ${ }^{(d)}$ Ghosh \& Soundararajaperumal (1992).

where $S$ and $H$ are in counts $\mathrm{s}^{-1}$. We searched for correlations using Pearson's product moment correlation coefficient $r$, defined as

$r=\frac{1}{n-1} \sum_{i=1}^{n}\left(\frac{x_{i}-\bar{x}}{\sigma_{x}}\right)\left(\frac{y_{i}-\bar{y}}{\sigma_{y}}\right)$,

where $x_{i}$ and $y_{i}$ are the values of the two data-sets we are comparing (namely the HR and the flux or the time), $n$ the size of the samples, $\bar{x}$ and $\bar{y}$ are the averages of the data-sets, and $\sigma_{x}$ and $\sigma_{y}$ are their standard deviations. In order to calculate the probability the null hypothesis ( $r=0$, namely no correlations) is true, we used the Fisher transformation

$F(r)=\frac{1}{2} \log \frac{1+r}{1-r}$,

from which we calculated $z=\sqrt{n-3} F(r)$, a $z$-score of $r$, from which we derived the probability $p$. We rejected the null hypothesis for $p<5 \%$.

The hardness ratio of ESO 383-18 is not constant $\left(\chi^{2}=34\right.$ for 70 d.o.f., $p=0.03 \%$ ), but does not show any significant correlation with time $(r=-0.3, p=99 \%)$ or flux $(r=0.05, p=$ $65 \%$ ). This lack of correlations might imply that the observed spectral variability on this time-scale is not due to absorbing material.
During the 2005 observation of ESO 140-43 the hardness ratio varied ( $\chi^{2}=188$ for 107 d.o.f., $p<0.01 \%$ ), while it is consistent with no variability during the 2006 observation $\left(\chi^{2}=\right.$ 24.3 for 99 d.o.f., $p \simeq 100 \%$ ). The 2005 observation does not show any correlation of the hardness ratio with flux $(r=0.26$, $p=61 \%)$ or time $(r=-0.55, p=99.9 \%)$, which indicates that changes in the absorbing material are not a likely explanation for the spectral variability on the $200 \mathrm{~s}$ time-scale. A strong variation of the average value of HR appears between the 2005 $\left(\overline{\mathrm{HR}^{1}}=-0.186 \pm 0.003\right)$ and the $2006\left(\overline{\mathrm{HR}^{2}}=+0.53 \pm 0.02\right)$ observations, consistent with the observed spectral variation.

\subsection{Optical and UV variability}

We analyzed the optical and UV emission of ESO 140-43 at the time of the X-ray observations with XMM-Newton/OM and Swift/UVOT data. In Table 7 the values of optical and ultraviolet magnitudes obtained are given. In the $V, B, U$, and $U V W I$ band the fluxes obtained by the two XMM-Newton observations are consistent, while in the $U V M 2$ band the source varied by about $\triangle U V M 2 \simeq 0.5 \mathrm{mag}$. UVOT measured the flux only in the $U V W 1$ and $U V M 2$ bands, and registered strong flux variations in $U V M 1$ with respect to the $\mathrm{OM}$ observations $(\triangle U V W 1 \simeq 0.9 \mathrm{mag}$ ), while the $U V M 2$ flux remained constant. 

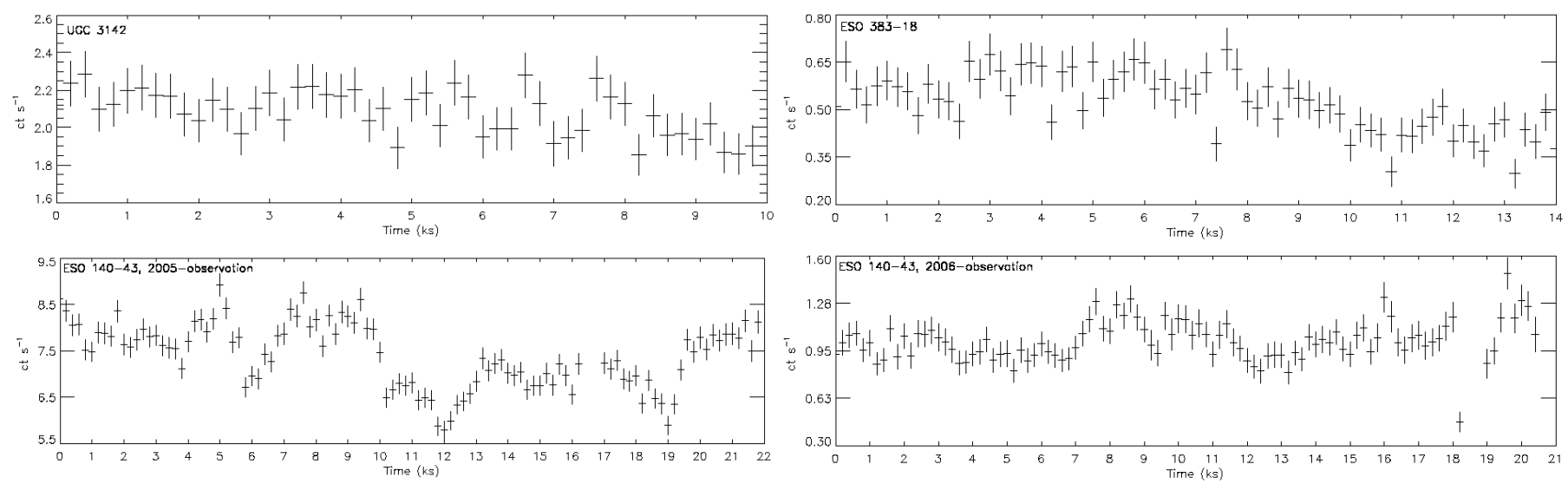

Fig. 11. EPIC PN light curves binned to $200 \mathrm{~s}$ for UGC 3142 (top left panel), ESO 383-18 (top right panel) and the 2005 (bottom left panel) and the 2006 observation of ESO 140-43 (bottom right panel).

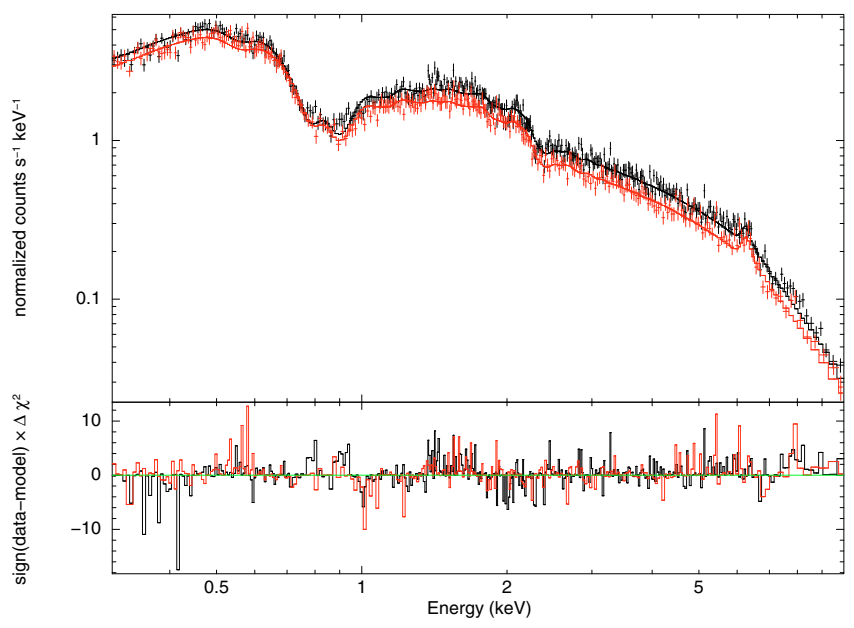

Fig. 12. EPIC/PN spectra of different time intervals according to the low flux state and the high flux state (in black) during the 2005-observation of ESO 140-43. The model is the best model obtained in Sect. 3.3, with all parameters fixed to the best model of this observation, except the covering factor of the LIA and the normalization.

\section{Discussion}

In this work we presented two AGN showing evidence for clumpy neutral absorbers and one showing evidence for clumpy ionized absorbers. We discuss these two cases separately, and then together in the light of the soft X-ray excess.

\subsection{UGC 3142 and ESO 383-18}

Recent results (e.g., Risaliti et al. 2009; Bianchi et al. 2009) show that clumpy absorbers can explain variability in AGN. Both Seyfert 1 (i.e. Guainazzi et al. 1998; Puccetti et al. 2007; Grupe et al. 2007) and Seyfert 2 (Risaliti et al. 2002) have been proven to show partial or total occultation events. In particular several Seyfert 2 have been observed changing state, switching from Compton-thin to reflection-dominated or vice versa (Matt et al. 2009). In their recent work Bianchi et al. (2009) propose a model consisting of three absorbers/emitters, not necessarily coexisting or observable in all the sources. These three components are a Compton-thick torus (at about parsec distance), a Compton-thin absorber $\left(N_{\mathrm{H}} \sim 10^{22} \mathrm{~cm}^{-2}\right)$ at much larger scales, likely associated to dust lanes, which completely or partially obscures the broad line region (BLR), and clouds at around the distance of the BLR. Recent evidence of clumpy structures in the torus has been found from Spitzer/IRS spectra (Mor et al. 2009). A clumpy moving absorber could also explain the optical change in the Seyfert type observed in some AGN (e.g., in Cohen et al. 1986; Aretxaga et al. 1999).

Both UGC 3142 and ESO 383-18 spectra show what at first sight appears to be a soft X-ray excess, though our analysis shows that the best fit to the data is obtained by a power law absorbed by two clumpy neutral absorbers. For the Seyfert 1 UGC 3142 we found that the two absorbers have column densities of the order of $10^{22} \mathrm{~cm}^{-2}$, and covering fractions of about $92 \%$ and $60 \%$. For the Seyert 2 ESO 383-18 the column densities are higher $\left(\simeq 10^{23} \mathrm{~cm}^{-2}\right)$, as expected from its optical classification, with covering fractions of about $97 \%$ and $86 \%$. The intrinsic absorption of the Seyfert 1 galaxy UGC 3142 is above the dividing line of $10^{22} \mathrm{~cm}^{-2}$, which usually points to a type 2 AGN. It has to be taken into account though that the transition between absorbed and unabsorbed sources is smooth, and that not all type 1 AGN necessarily exhibit low absorption (Awaki et al. 1991). In addition, Seyfert 2 galaxies like NGC 3147 and NGC 4698 show no intrinsic absorption (Pappa et al. 2001). The INTEGRAL AGN catalog (Beckmann et al. 2009) contains 9 type 1 AGN with $N_{\mathrm{H}}>10^{22} \mathrm{~cm}^{-2}$ and 10 Seyfert 2 with $N_{\mathrm{H}}<10^{22}$ out of 154 Seyfert galaxies with known intrinsic absorption.

Following the model of Bianchi et al. (2009), the partially covering absorbers seen in ESO 383-18 and UGC 3142 imply a certain degree of clumpiness in the BLR or in the torus, or dust lanes. An additional component could be represented by a clumpy neutral (or weakly ionized) disk wind, crossing the line of sight. These outflows, originating in the accretion flow, are predicted by hydrodynamic simulations of accretions disks (e.g., Schurch et al. 2009). According to this, in the case of ESO 38318 we could picture the absorption as due to a clumpy torus, plus a disk wind or Compton-thin dust lanes with a smaller column density, while for UGC 3142 we could make the conjecture that the observed absorbers are part of a disk wind or of the BLR.

Variations in the partial covering absorbers have been shown to cause strong spectral and flux variability in AGN (e.g., 1H 0707-495, Gallo et al. 2004). ESO 383-18 shows variability in both flux (of $\simeq 14 \%$ ) and hardness ratio, but, because the hardness ratio is not correlated with time or flux, we exclude that the changes on the $200 \mathrm{~s}$ time-scale could be caused by changes in the absorbers. The observed spectral variability is 
probably due to changes of the continuum. In the EPIC spectrum of ESO 383-18 we detected a feature that could be associated with the OVIII Ly $\alpha$ emission line, but due to the low significance of the data this has to be considered as a tentative identification. The OVIII Ly $\alpha$ appears to be a common feature in absorbed AGN, and it was detected in 30 of the 69 nearby AGN studied by Guainazzi \& Bianchi (2007).

For both objects we cannot confirm or rule out the hypothesis that the neutral matter responsible for clumpy absorption is part of a disk wind. Indeed no blue-shifted absorption lines, which would indicate out-flowing matter, are detected. We can nevertheless safely state that the iron $\mathrm{K} \alpha$ line in both objects is not produced in out-flowing material, because the line's centroid is consistent with the redshift of the host galaxy. The non-detection of a reflection hump in the two AGN despite narrow neutral iron lines is probably due to the low significance of IBIS/ISGRI and BAT data for ESO 383-18, and might be hidden by the strong variability in hard X-rays for UGC 3142.

It should be taken into account that partial-covering models are indistinguishable from Thomson scattering models (Turner $\&$ Miller 2009), thus an alternative explanation could be that part of the continuum has been scattered energy-independently and that the absorbers are not partially covering the X-ray source.

\subsection{ESO $140-43$}

Warm absorbers have been found to be a common characteristic of Seyfert 1 AGN. In a study of 15 Seyfert 1 McKernan et al. (2007) found that 10 sources of their sample present out-flowing photo-ionized absorbers, with velocities in the range $v \sim 0-2000 \mathrm{~km} \mathrm{~s}^{-1}$, column densities of about $N_{\mathrm{H}} \sim$ $10^{20-23} \mathrm{~cm}^{-2}$ and ionization parameters in a wide range of $\xi \sim$ $1-10^{4} \mathrm{erg} \mathrm{cm} \mathrm{s}^{-1}$. Several of these absorbers show multiple ionization and kinematics components.

The Seyfert 1 galaxy ESO 140-43 shows a very complex X-ray spectrum, which can be modeled with a power law absorbed by three layers of partially covering ionized material, plus a Gaussian iron $\mathrm{K} \alpha$ line at the same redshift as the host galaxy, and a weak reflection hump. The XMM-Newton/RGS data of one of the two observations of ESO 140-43 were analyzed by Jiménez-Bailón et al. (2008), who also detected three warm absorbers, and, contrarily to what we obtained here, also found an additive cold absorber. The photon index they obtained $(\Gamma=2.0 \pm 0.1)$ is consistent with what was found here $(\Gamma \simeq 1.9)$. The results we obtained agree with the current picture of multi-structure warm absorbers of Seyfert 1 AGN, in which several zones of warm material with different ionization states are observed. Examples are the Seyfert 1 galaxies NGC 3783 (Netzer et al. 2003; Krongold et al. 2003) and MCG 6-30-15 (McKernan et al. 2007), the last of these showing evidence for a similar complex absorber consisting of three zones of ionized gas. The Seyfert 1 NGC 3516 (Turner et al. 2005) shows evidence for a warm absorber covering about $50 \%$ of the continuum source, with the variation of its covering factor being used to explain the observed spectral variability (Turner et al. 2008). A powerful tool for the diagnostic of the physical conditions of ionized material in AGN is the study of absorption lines below $2 \mathrm{keV}$ (e.g., Porquet \& Dubau 2000). The RGS spectrum of ESO 140-43 shows several absorption lines due to H- and He-like elements. These lines are a common characteristics of Seyfert 1 galaxies (e.g., Longinotti et al. 2008; Armentrout et al. 2007; Nucita et al. 2010) and are also found in several absorbed AGN (Bianchi \& Guainazzi 2007). The best X-ray model, the absorption lines due to ionized material and the strong variability of ESO 140-43 imply ionized clumpy absorbers, which cross the line of sight toward the central engine producing the X-ray continuum.

Due to the strong spectral and flux variability on a timescale of months, the three clumpy warm absorbers detected in the spectrum of ESO 140-43 could be part of a disk wind, as seen in several other Seyfert 1 (e.g., Turner et al. 2008). But because we do not detect any evidence of outflows, we cannot confirm this hypothesis. Possible explanations of the mechanism by which the out-flowing gas is accelerated are thermal winds (Krolik \& Begelman 1986), radiation pressure (Murray et al. 1995), or magnetically driven winds (Bottorff et al. 2000). In the disk-wind scenario the observed variability could be due to changes in the physical conditions of the disk, or caused by interaction of a wind with other material. The detection of UV variability, considering that the optical/UV emission is probably disk dominated, supports the assumption that the observed variability is due to changes in the physical conditions of the accretion disk. An alternative scenario could be that the three warm absorbers are part of the BLR, and we observe occultations as seen for NGC 1365 (Risaliti et al. 2007). Both EPIC observations of ESO 140-43 show a narrow neutral iron line and no evidence of a broad component. The line flux varied by about $45 \%$ between the two observations, comparable with the variation of the continuum. As all the absorbers we detected are ionized, we can conclude that the neutral iron line and the reflection hump (which is also due to reflection on neutral matter) are produced by material which is not located in the line of sight. Possible locations for the line emitting region might be the torus or the outer part of the accretion disk.

Flux (of $\simeq 9 \%$ ) and spectral variability on the time-scale of $200 \mathrm{~s}$ is observed in the light curves obtained from both EPIC observations, but the lack of significant correlations between the hardness ratio and the flux indicates that the absorbers do not strongly influence the variability on this time-scale, and that variations in the continuum probably play a more important role. On longer time-scales (kiloseconds) the influence of the absorbers starts to be more evident. A variation of the covering factor of the LIA is indeed necessary to model the change between the high and the low flux state of the 2005 observation. This result agrees with the strong influence of the absorbers on the observed spectral variability of ESO 140-43.

The location and geometry of the ionized gas are still debated, but a rough estimate of the upper limit on its distance to the ionizing source can be given (Turner et al. 2008) by

$$
r \lesssim \frac{L}{\xi N_{\mathrm{H}}} .
$$

Using this relation, and considering the values obtained by the best fitting models, we estimated upper limits for the distance to the central engine of the three ionized absorbers. For the 2005 observation we obtained $r_{\mathrm{HIA}} \lesssim 2.9 \mathrm{pc}, r_{\mathrm{IIA}} \lesssim 6.6 \mathrm{pc}$ and $r_{\text {LIA }} \lesssim 2.5 \mathrm{kpc}$, while for the 2006 observation $r_{\mathrm{HIA}} \lesssim 0.05 \mathrm{pc}$, $r_{\text {IIA }} \lesssim 2 \mathrm{pc}$ and $r_{\text {LIA }} \lesssim 23 \mathrm{kpc}$. The luminosity was calculated extrapolating the power law continuum into the $5 \mathrm{eV}-300 \mathrm{keV}$ band. These values are not constraining, except for the HIA, which appears to be significantly closer to the central engine than the torus. The value of the HIA obtained from the 2006 observation is similar to what has been found for the highest ionization absorber of NGC 3783 (Reeves et al. 2004). All the upper limits found here are well above the value of the inner radius of the BLR of ESO 140-43 found by Padovani \& Rafanelli (1988), 
$r_{\text {in }}^{\text {BLR }} \simeq 8.9 \times 10^{-3} \mathrm{pc}$. This result does not allow us to exclude the BLR to be, at least partially, responsible for the absorption.

\subsection{Clumpy absorbers and the soft excess}

The nature of the soft X-ray excess of AGN has remained a mystery for the past two decades. Several explanations have been proposed, here we focus in particular on the absorption models (e.g., Gierlinski \& Done 2004; Guainazzi et al. 2005; Schurch \& Done 2006). In the velocity-smeared, partially ionized absorption scenario (Gierlinski \& Done 2004; Schurch \& Done 2006) the observed soft excess is related to atomic transitions, in particular to the large increase in opacity in partially ionized material, due to lines and edges of ionized OVII, OVIII and iron at $\sim 0.7 \mathrm{keV}$. In this model, the partially ionized material is thought to be a disk wind with a complex velocity structure, which makes it difficult to resolve the atomic features. Schurch \& Done (2008) noted that magnetic driving is likely the only mechanism that could achieve the high velocities required. While we do not detect any evidence of high velocities components in the absorbing medium, we found that the absorbers are clumpy, consistent with the assumptions of Schurch \& Done (2008).

Warm absorbers are likely to be the main cause for the soft X-ray excess observed in many AGN (Turner et al. 2009), in this context ESO 140-43, with its three partially covering ionized absorbers, gives further evidence on how absorption can explain the soft excess in some Seyfert galaxies. Neutral absorbers can also play a significant role in the observed soft excess of AGN (Matt et al. 2001; Guainazzi et al. 2005). In the clumpy neutral absorber scenario adopted here, the X-ray source is not completely covered by the absorbers, and this allows part of the flux below $2 \mathrm{keV}$ to leak out. Considering an absorber totally obscuring the primary X-ray emission (i.e. the power law continuum), we over-estimate the absorption below $2 \mathrm{keV}$, which then results in an apparent excess over the model.

Although for the three AGN presented here the absorption model well fits the observed soft X-ray excess, from previous studies it is obvious that not one model alone can account for what is observed in the spectra of many sources. Guainazzi et al. (2005) showed in a sample of 26 X-ray spectra of Seyfert 2 galaxies that 18 were best represented by an additional component $(k T=0.1-1.5 \mathrm{keV}), 4$ objects required clumpy absorbers but no additional component, and 4 did not require any addition to the absorbed power law model.

It is remarkable that the type of model which fits the data best does not depend on the source type. There are type 1 and type 2 objects best modeled by an absorbing scenario (e.g. this work), and there are Seyfert 1 and Seyfert 2 galaxies for which a reflection model (e.g., Petrucci et al. 2007; Middleton et al. 2009; Wilkes et al. 2008), or an additional component like a cool corona (e.g., Dewangan et al. 2007; Guainazzi et al. 2005), represents the best model. This would indicate that the effects causing the soft excess do not depend on source type and inclination angle of the accretion disk. On the other hand, Pounds \& Wilkes (2007) pointed out that the effect of the soft excess is more pronounced in type 1 than in type 2 objects, and that a Seyfert 1.5 like 2MASS 0918+2117 indeed shows an intermediate excess. This would point to an origin of the soft excess far within the absorbing material responsible for the type 1 / type 2 distinction, and would support the hypothesis of Bianchi et al. (2009) that the observed X-ray spectra are influenced by several different absorbers. Larger well-defined samples, as presented e.g. in Guainazzi et al. (2005), with a consistent modeling approach are necessary though to verify this hypothesis.

\section{Summary and conclusions}

We performed the first detailed X-ray analysis of the three Seyfert galaxies ESO 140-43, ESO 383-18, and UGC 3142 using XMM-Newton high resolution data in combination with INTEGRAL/IBIS and Swift BAT/XRT data. The spectra of the three AGN presented indeed show what looks like a soft excess at energies $E<2 \mathrm{keV}$, but which we have shown is due to complex absorption, without any additive emission to the power law continuum.

The $0.3-10 \mathrm{keV}$ spectrum of the three Seyfert galaxies is well fit by a power law, absorbed by two partially covering neutral absorber for UGC 3142 and ESO 383-18, and by three clumpy ionized absorbers in the case of ESO 140-43. ESO 14043 also shows evidence for a reflection hump.

The absorbing medium seen in ESO 383-18 could be a clumpy torus, plus a disk wind or Compton thin dust lanes. For UGC 3142 the observed absorbers might be part of a disk wind, or of the BLR. The variability of the flux and of the hardness ratio observed in the light curve of ESO 383-18 is not explainable by variations of the absorbers, and is likely related to changes of the continuum.

The absorbers of ESO 140-43 have different values of column densities and ionization states, according to the current picture of warm absorbers. Strong flux and spectral (accounted for by changes in the three warm absorbers) variability was detected between ROSAT, EXOSAT, and the two XMM-Newton observations of ESO 140-43. The spectral variability is likely due to the fact that the clumpy absorbers are moving, either as a part of a disk wind or of the BLR. During the 2005-observation of ESO 140-43 variability of the covering factor of the LIA is detected on a time-scale of kilo-seconds, while on shorter timescales the observed spectral variability cannot be explained by changes in the absorbers. Thus the effects of absorption on the variability are observable only on time-scales longer than few kilo-seconds, and are probably hidden by changes of the continuum on shorter time-scales.

We reported the first detection of the iron $K \alpha$ line in the spectra of ESO 383-18 and UGC 3142, and confirmed its presence in the spectrum of ESO 140-43. We tentatively associate an excess around $0.6 \mathrm{keV}$ in the spectrum of ESO 383-18 with OVIII Ly $\alpha$ emission. More sensitive data will be necessary to confirm or rule out the presence of this line. The detection of the iron $K \alpha$ line, but not of any additive sign of reflection (i.e. a reflection hump) for ESO 383-18 and UGC 3142 is probably due to the low significance of IBIS/ISGRI data and the hard X-rays variability, respectively.

In general there does not seem to be a common model explaining the soft X-ray excess in all the observed cases. Rather it is likely that several components play a role, like reflection processes, complex absorption, and additional thermal emission. Not all these components are observed in all objects, and it remains to be clarified whether the source type and inclination angle of the accretion disk has an influence on the type of soft excess observable in the X-ray spectra. It is anyway likely that some of the reported soft X-ray excesses in Seyfert galaxies are indeed caused by clumpy (neutral or ionized) absorbers, which allow a portion of the primary flux emitted by the central X-ray source to reach the observer directly. Thus the soft excess might simply be related at least in some cases to an incorrect absorption model, which over-estimates the absorbed flux below $2 \mathrm{keV}$.

New broad band X-ray observations of these three Seyfert galaxies, e.g. by Suzaku, would be fundamental to probe the hypothesis that the absorption is responsible for the month scale 
variability, and to give better limits to the sizes and locations of the absorbers.

Acknowledgements. We would like to thank Simona Soldi, Chin-Shin Chang, Carla Baldovin and Marc Türler for their useful comments on the work, Andrew Taylor for reading and correcting the manuscript, and Tim Kallman for his help with XSTAR. This research has made use of the NASA/IPAC Extragalactic Database (NED) which is operated by the Jet Propulsion Laboratory, of data obtained from the High Energy Astrophysics Science Archive Research Center (HEASARC), provided by NASA's Goddard Space Flight Center, and of the SIMBAD Astronomical Database which is operated by the Centre de Données astronomiques de Strasbourg. We would also like to thank the anonymous referees for their comments, which helped to improve this paper.

\section{References}

Abrassart, A., \& Czerny, B. 2000, A\&A, 356, 475

Antonucci, R. R. J. 1993, ARA\&A, 31, 473

Armentrout, B. K., Kraemer, S. B., Turner, T. J. 2007, ApJ, 665, 237

Arnaud, K. A. 1996, in: Astronomical Data Analysis Software and Systems V, ed. G. Jacoby, \& J. Barnes, ASP Conf. Ser., 101, 17

Aretxaga, I., Joguet, B., Kunth, D., Melnick, J., \& Terlevich, R. J. 1999, ApJ, 519, L123

Awaki, H., Koyama, K., Inoue, H., \& Halpern, J. P. 1991, PASJ, 43, 195

Bassani, L., Molina, M., Malizia, A., et al. 2006, ApJ, 636, 65

Beckmann, V., Gehrels, N., Favre, P., et al. 2004, ApJ, 614, 641

Beckmann, V., Petry, D., Weidenspointner, G. 2007, ATel, 1264

Beckmann, V., Soldi, S., Ricci, C., et al. 2009, A\&A, 505, 417

Bianchi, S., \& Guainazzi, M. 2007, AIP Conf. Proc., 924, 822

Bianchi, S., Piconcelli, E., Chiaberge, M., et al. 2009, ApJ, 695, 781

Blustin, A. J., Page, M. J., Fuerst, S. V., et al. 2005, A\&A, 431, 111

Blustin, A. J., Kriss, G. A., Holczer, T., et al. 2007, A\&A, 466, 107

Boller, T., Meurs, E. I. A., Brinkmann, W., et al. 1992, A\&A, 261, 57

Bottorff, M. C., \& Ferland, G. J. 2000, MNRAS, 316, 103

Burrows, D. N., Romano, P., Falcone, A., et al. 2005, SSRv, 120, 165

Caroli, E., Stephen, J. B., Di Cocco, G., Natalucci, L., \& Spizzichino, A. 1987, Space Sci. Rev., 45, 349

Chevallier, L., Collin, S., Dumont, A.-M., et al. 2006, A\&A, 449, 493

Cohen, R. D., Puetter, R. C., Rudy, R. J., et al. 1986, ApJ 311, 135

Courvoisier, T. J.-L., \& Türler, M. 2005, A\&A, 444, 417

Courvoisier, T. J.-L., Walter, R., Beckmann, V., et al. 2003, A\&A, 411, 53

Cusumano, G., La Parola, V., Segreto, A., et al. 2010, A\&A, 510, A48

Decarli, R., Dotti, M., Fontana, M., \& Haardt, F. 2008, MNRAS, 386, L15

Dewangan, G. C., Griffiths, R. E., Dasgupta, S., \& Rao, A. R. 2007, ApJ, 671, 1284

Dickey, J. M., \& Lockman, F. G. 1990, ARA\&A, 28, 215

Done, C., \& Nayakshin, S. 2007, MNRAS, 377, L59

Done, C., Mulchaey, J. S., Mushotzky, R. F., \& Arnaud, K. A. 1992, ApJ, 395, 275

Gallo, L. C., Tanaka, Y., \& Boller, T. 2004, MNRAS, 353, 1064

Gehrels, N., Chincarini, G., Giommi, P., et al. 2004, ApJ, 611, 1005

George, I. M., Turner, T. J., Mushotzky, R., et al. 1998, ApJ, 503, 174

Ghosh, K. K., \& Soundararajaperumal, S. 1992, MNRAS, 259, 175

Gierlinski, M., \& Done, C. 2004, MNRAS, 349, L7

Goldwurm, A., David, P., Foschini, L., et al. 2003, A\&A, 411, L223

Grupe, D., Komossa, S., \& Gallo, L. C. 2007, ApJ, 668, L111

Guainazzi, M., \& Bianchi, S. 2007, MNRAS, 374, 1290

Guainazzi, M., Matt, G., Antonelli, L. A., et al. 1998, MNRAS, 298, 824

Guainazzi, M., Matt, G., \& Perola, G.-C. 2005, A\&A, 444, 119

Guilbert, P. W., \& Rees, M.-J. 1988, MNRAS, 233, 475

Haardt, F., \& Maraschi, L. 1991, ApJ, 380, L51

Haardt, F., \& Maraschi, L. 1993, ApJ, 413, 507

Halpern, J. P. 1984, ApJ, 281, 90

Holt, S. S., Mushotzky, R. F., Boldt, E. A., et al. 1980, ApJ, 241, L13
Ishibashi, W., \& Courvoisier, T. J.-L. 2009, A\&A, 495, 113 Jansen, F., Lumb, D., Altieri, B., et al. 2001, A\&A, 365, L1 Jiménez-Bailón, E., Guainazzi, M., Matt, G., et al. 2008, RMxAC, 32, 131 Kallman, T. R., \& Bautista, M. 2001, ApJS, 133, 221

Kallman, T. R., Liedahl, D., Osterheld, A., Goldstein, W., \& Kahn, S. 1996, ApJ, 465, 994

Kaspi, S., Brandt, W. N., George, I. M., et al. 2002, ApJ, 574, 643

Kinney, A. L., Schmitt, H. R., Clarke, C. J., et al. 2000, ApJ, 537, 152

Krivonos, R., Revnivtsev, M., Lutovinov, A., et al. 2007, A\&A, 475, 775

Krolik, J. H., \& Begelman, M. C. 1986, ApJ, 308, L55

Krongold, Y., Nicastro, F., Brickhouse, N. S., et al. 2003, ApJ, 597, 832

Longinotti, A. L., Nucita, A. A., Santos, L. M., \& Guainazzi, M. 2008, A\&A, 484, L311

Longinotti, A. L., Costantini, E., Petrucci, P. O., et al. 2010, A\&A, 510, A92

Magdziarz, P., \& Zdziarski, A. A. 1995, MNRAS, 273, 873

Markowitz, A., Edelson, R., \& Vaughan, S. 2003, ApJ, 598, 935

Marshall, F., Boldt, E., Halt, S., et al. 1979, ApJS, 40, 657

Mason, K. O., Breeveld, A., Much, R., et al. 2001, A\&A, 365, L36

Matt, G., Guainazzi, M., Perola, G. C., et al. 2001, A\&A, 377, L31

Matt, G., Bianchi, S., De Rosa, A., et al. 2006, A\&A, 445, 451

Matt, G., Bianchi, S., Awaki, H., et al. 2009, A\&A, 496, 653

McKernan, B., Yaqoob, T., \& Reynolds, C. S. 2007, MNRAS, 379, 1359

Middleton, M., Done, C., Ward, M., Gierliński, M., \& Schurch, N. 2009, MNRAS, 394, 250

Mor, R., Netzer, H., \& Elitzur, M. 2009, ApJ, 705, 298

Murray, N., Chiang, J., Grossman, S. A., \& Voit, G. M. 1995, ApJ, 451, 498

Nandra, K., Fabian, A. C., George, I. M., et al. 1993, MNRAS, 260, 540

Nandra, K., George, I. M., Mushotzky, R., Turner, T. J., \& Yaqoob, T. 1997, ApJ, 476, 70

Netzer, H., Kaspi, S., Behar, E., et al. 2003, ApJ, 599, 933

Nucita, A. A., Guainazzi, M., Longinotti, A. L., et al. 2010, A\&A, 515, A47

Padovani, P., \& Rafanelli, P. 1988, A\&A, 205, 53

Pan, H. C., Stewart, G. C., \& Pounds K. A. 1990, MNRAS, 242, 177

Pappa, A., Georgantopoulos, I., Stewart, G. C., \& Zezas, A. L. 2001, MNRAS, 326, 995

Petrucci, P. O., Ponti, G., Matt, G., et al. 2007, The Multicolored Landscape of Compact Objects and Their Explosive Origins, AIPC, 924, 583

Porquet, D., \& Dubau, J. 2000, A\&AS, 143, 495

Pounds, K. A., \& Wilkes, B.-J. 2007, MNRAS, 380, 1341

Pounds, K. A., Nandra, K., Fink, H. H., \& Makino, F. 1994, MNRAS, 267, 193

Piconcelli, E., Jiménez-Bailón, E., Guinazzi, M., et al. 2005, A\&A, 432, 15

Puccetti, S., Fiore, F., Risaliti, G., et al. 2007, MNRAS, 377, 607

Reeves, J. N., Nandra, K., George, I. M., et al. 2004, ApJ, 602, 648

Reichert, G. A., Mushotzky, R. F., Petre, R., \& Holt, S. S. 1985, ApJ, 296, 69

Reynolds, C. S. 1997, MNRAS, 286, 513

Risaliti, G., Elvis, M., \& Nicastro, F. 2002, ASPC, 258, 81

Risaliti, G., Elvis, M., Fabbiano, G., et al. 2006, ESASP, 604, 655

Risaliti, G., Elvis, M., Fabbiano, G., et al. 2007, ApJ, 659, L111

Risaliti, G., Salvati, M., Elvis, M., et al. 2009, MNRAS, 393, 1

Roming, P. W. A., Kennedy, T. E., Mason, K. O., et al. 2005, SSRv, 120, 95

Saxton, R. D., Turner, M. J. L., Williams, O. R., et al. 1993, MNRAS, 262, 63

Schurch, N. J., \& Done, C. 2006, MNRAS, 371, 81

Schurch, N. J., \& Done, C. 2008, MNRAS, 386, L1

Schurch, N. J., Done, C., \& Proga, D. 2009, ApJ, 694, 1

Soldi, S., Beckmann, V., Bassani, L., et al. 2005, A\&A, 444, 431

Steenbrugge, K. C., Kaastra, J. S., Crenshaw, D. M., et al. 2005, A\&A, 434, 569

Strüder, L., Briel, U., Dennerl, K., et al. 2001, A\&A, 365, L18

Titarchuk, L. 1994, ApJ, 434, 313

Tueller, J., Baumgartner, W. H., Markwardt, C. B., et al. 2010, ApJS, 186, 378

Turner, T. J., \& Miller, L. 2009, A\&ARv, 17, 47

Turner, T. J., Nandra, K., George I. M., Fabian, A. C., \& Pounds, K. 1993, ApJ, 419, 127

Turner, M. J. L., Abbey, A., Arnaud, M., et al. 2001, A\&A, 365, L27

Turner, T. J., Reeves, J. N., Kraemer, S. B., \& Miller, L. 2008, A\&A, 483, 161

Vaughan, S., Edelson, R., Warwick, R. S., \& Uttley, P. 2003, MNRAS, 345, 1271

Wilkes, B.-J., Pounds, K.-A., \& Schmidt, G.-D. 2008, ApJ, 680, 110 